Pacific Journal of Mathematics

EXTENSIONS OF GENERALIZED HOMOLOGY THEORIEs 


\title{
EXTENSIONS OF GENERALIZED HOMOLOGY THEORIES
}

\author{
FRIEDRICH W. BAUER \\ James Dugundji in memoriam
}

\begin{abstract}
Relations between different kinds of homology theories on the category Com (of compacta) resp. the related strong shape category $\overline{\mathbf{C o m}}$ are studied. In particular homology theories satisfying a clusteraxiom (as for example the strong shape homology $\bar{E}_{*}$ with coefficients in a spectrum $\underline{E}$, for a restricted class of spectra being defined on the category of finite dimensional compacts) allow interesting characterizations. As an application this provides new proofs of classical theorems concerning Steenrod-Sitnikov homology theories, including a result on the BrownDouglas-Fillmore homology $\varepsilon_{*}$.
\end{abstract}

0. Let $\underline{E}$ be any $\mathrm{CW}$-spectrum and $\underline{E}_{*}$ the corresponding homology theory [1] on the category $\underline{P}_{0}$ of based, finite CW-spaces.

We are concerned with the following questions:

(1) Can $\underline{E}_{*}$ be extended over the categories Com (= based compact metric spaces) or alternatively over $\underline{K}$, the strong shape category?

(2) Under what circumstances is such an extension uniquely determined?

These two problems are both extension problems. The first one deals with the extension of $\underline{E}_{*}$ itself, while the second one requires the extension of a given isomorphism between two homology theories.

The solution of both problems deserves interest because it turns out that numerous problems in topology and analysis can be reduced to (1) or (2).

There are for example two "natural" (not in the sense of "canonical") extensions of complex $K$-homology $\underline{B U}_{*}$ over a category containing $\underline{P}_{0}$ : (1) The homology theory $\overline{\mathrm{BU}}_{*}$ defined on $\overline{\mathrm{Com}}$ (the subcategory of $\underline{\bar{K}}$, determined by compact metric spaces) and (2) the Brown-Douglas-Fillmore homology theory $\varepsilon_{*}$ defined on Com ([6], [8], [9]) by purely func-

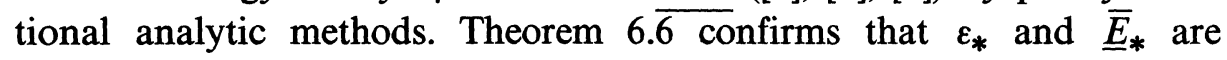
naturally isomorphic for finite dimensional spaces in Com.

D. Edwards and H. Hastings [7] as well as the authors of [8] also dealt with the problem of establishing generalized, so called Steenrod homology theories. The relation between the homology theory ${ }^{s} \underline{E}_{*}$ of [8] and our $\underline{E}_{*}$ 
is the subject of [3], while Theorem 5.7 of the present paper confirms the existence of an isomorphism between ${ }^{s} \underline{E}_{*}$ and the Edwards-Hastings kind of Steenrod extensions of a homology theory (cf. remark at the end of §7). The strong shape category $\underline{\bar{K}}$ (resp. the homotopy category $\underline{K}_{h}$ (which is used in this paper (cf. §7) differs formally from that of [7]. However Ju. Lisica [11] established in the meantime the equivalence of $\underline{K}_{h}$ with the strong shape category s-Sh of [7]. This clarifies the relations between different concepts of Steenrod homology theories, shape homology theories resp. the related strong shape categories, which were introduced independently but approximately at the same time.

J. Milnor characterized in [12] ordinary homology theories (i.e. those with $\underline{E}=K(G)$, an Eilenberg-Mac Lane spectrum) on Com axiomatically. We get back this result in the following form (Theorem 6.4.): $\overline{K(G)}$ * on Com, is up to an isomorphism, uniquely determined by the EilenbergSteenrod axioms with a strong excision axiom ( $\$ 5 \mathrm{~A} 2)$ ) and the clusteraxiom ( $\$ 5 \mathrm{~A} 1)$ ). This is a corollary of a more general result (Corollary 5.7) which deals with arbitrary $\mathrm{CW}$-spectra $\underline{E}$ having the property that a clusteraxiom for $\bar{E}_{*}$ on compact metric spaces is valid. Because the spectrum BU (the classifying spectrum of complex $K$-theory [1]) turns out to be, at least for finite dimensional compact metric spaces (Proposition 4.4 ), of this kind, the above mentioned result on $\varepsilon_{*}$ is another corollary of this assertion.

The extension problem can be approached from a somewhat different point of view leading to the concept of shape singular homology theory with coefficients in a $C W$ spectrum $\underline{E}(\S \S 1-3)$ : Let $\underline{\bar{K}}$ be the strong shape category with arbitrary based spaces. Does there exist an extension of $\underline{E}_{*}$ over $\underline{\bar{K}}_{h}$ (the corresponding homotopy category) having the following two properties:

(1) Every weak homotopy equivalence in $\underline{K}_{h}$ (Definition 1.2) induces an isomorphism of the homology groups.

(2) The extended homology theory has compact carrier (Definition $1.7) ?$

Theorem 1.6 provides us with an affirmative answer to this question. Moreover the extension (denoted by $\underline{E}_{*}$ ) is unique up to an isomorphism (Theorem 3.1).

Even for compact metric $X$ we can in general not expect to obtain an isomorphism $\underline{E}_{*}(X) \approx \underline{E}_{*}(X)$. However one has for all $X$ an isomorphism (Theorem 6.1):

$$
\underline{\tilde{E}}_{*}(X) \approx \underline{E}_{*}(|\bar{S}(X)|)
$$


where $\bar{S}(X)$ denotes the shape-singular complex of the space $X$. So $\underline{E}_{*}$ reveals itself as shape singular homology. The question under what circumstances $\tilde{E}_{*}$ is isomorphic to $\bar{E}_{*}$ is settled in Theorem 6.1. This result in combination with Theorem 6.2 (asserting that Steenrod-Sitnikov homology groups $H_{*}^{S}(X ; G)$ being for all compact metric $X$ and all abelian groups $G$ naturally isomorphic to $\overline{K(G)} *(X)$ ) yields another proof of Theorem 7.7 in [2], claiming that $\overline{H_{*}^{S}(X ; S)}$ is naturally isomorphic to $H_{*}(|\bar{S}(X)| ; \mathbf{Z})$ for shape connected compacta $X$. It turns out that for $s$-continua, $\tilde{E}_{*}$ can be regarded as the appropriate generalization of Borel-Moore homology theory (cf. [10]) while $\underline{E}_{*}$ corresponds to Steenrod-Sitnikov homology theory. In a final section $(\$ 7)$ we indicate the construction of the shape category and of the shape homology in this category with coefficients in a CW-spectrum. This is done without going into the details, referring to the relevant expositions (cf. [4]). The present paper is very closely related (but independent of) [3], which has already appeared. Therefore [3] refers occasionally to the present paper (concerning some details of proofs).

1. The existence theorem. Unless stated otherwise, we denote in the first three sections by $\underline{K}$ the category Top ${ }_{0}$. We are working within the strong shape category $\underline{\bar{K}}$ resp. the associated homotopy category $\underline{K}_{h}$, whose construction is briefly recorded in §7. For more details see [3], [4].

1.1. Definition. A morphism $\bar{f} \in \underline{\bar{K}}(X, Y)=\underline{\bar{K}}\left(\left(X, x_{0}\right),\left(Y, y_{0}\right)\right)$ is called a weak homotopy equivalence whenever

$$
|\bar{S}(\bar{f})| \in \underline{\operatorname{Top}}_{0}(|\bar{S}(X)|,|\bar{S}(Y)|)
$$

is a homotopy equivalence, $\bar{S}()$ denoting the shape singular complex, cf. $\S 7$. Let $\mathfrak{S}_{*}=\left\{\mathscr{\mathfrak { B }}_{n}\right\}: \underline{\bar{K}}_{h} \rightarrow \underline{\mathrm{Ab}}^{\mathbf{Z}}$ (= category of Z-graded abelian groups) be a functor, then we introduce the following Whitehead axiom:

1.2. Definition. (W) Let $\bar{f} \in \underline{\bar{K}}(X, Y)$ be a weak homotopy equivalence, then $\mathfrak{S}_{*}(\bar{f})$ is an isomorphism.

1.3. Definition. A functor $\mathfrak{F}_{*}: \bar{K}_{h} \rightarrow \mathrm{Ab}^{\mathrm{z}}$ together with a natural

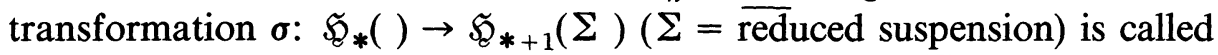
a shape singular homology theory $\mathfrak{S}_{*}=\left\{\mathfrak{S}_{n}, \sigma\right\}$ on $\underline{K}_{h}$ whenever $\mathfrak{H}_{*}$ satisfies the Whitehead axiom (W). We will during this and the two ensuing sections simply talk about a homology theory $\mathfrak{S}_{*}$. A natural transformation $\varphi: \mathfrak{S}_{*} \rightarrow \mathfrak{S}^{\prime}{ }_{*}$ between the functors $\mathfrak{S}_{*}, \mathfrak{S}^{\prime}{ }_{*}$ is called a 
transformation of homology theories whenever $\varphi$ commutes with the corresponding transformations $\sigma: \mathscr{S}_{*}() \rightarrow \mathscr{S}_{*+1}(\Sigma)$ resp. $\sigma^{\prime}: \mathscr{S}^{\prime}{ }_{*}() \rightarrow$ $\breve{S}^{\prime} *+1(\Sigma)$.

REMARK. Here we do not require that the natural transformation $\sigma$ is an isomorphism. This changes in $\$ 5$.

Let $\underline{P}_{1}\left(\underline{P}_{0}\right)$ be the category of (compact) CW-spaces, understood as a subcategory of $\underline{\bar{K}}$. Because of $7.1 \underline{P}_{1 h}$ is a full subcategory of $\bar{K}_{h}$. Our first aim is to extend a given homology theory $\mathfrak{H}_{*}: \underline{P}_{1 h} \rightarrow \underline{\mathrm{Ab}}$ over $\underline{K}_{h}$. We need ([2] Theorem 5.1.c).

1.4. Lemma. Let $X \in \underline{T o p}_{0}$ be any based space, then the natural transformation

$$
\bar{\omega}_{X} \in \underline{\bar{K}}(|\bar{S}(X)|, X)
$$

(cf. §7) is a weak homotopy equivalence. Every weak homotopy equivalence induces isomorphisms

$$
\bar{\pi}_{*}(\bar{f}) \quad\left(\text { with } \bar{\pi}_{n}\left(X, x_{0}\right)=\underline{K}_{h}\left(\left(S^{n}, *\right),\left(X, x_{0}\right)\right)\right) .
$$

Suppose that $X, Y \in \mathrm{Top}_{0}$ are s-connected (= shape connected, i.e. $\bar{\pi}_{0}(X)$ $\left.=\bar{\pi}_{0}(Y)=0\right)$; then also the converse holds.

Proof. The first part follows because $\left|\bar{S}\left(\bar{\omega}_{X}\right)\right|:|\bar{S}(|\bar{S}(X)|)| \rightarrow|\bar{S}(X)|$ is evidently (because everything happens in $\underline{P}_{1}$ ) a homotopy equivalence. Let $\bar{f} \in \underline{\bar{K}}(X, Y)$ be a weak homotopy equivalence. Then we have the commutative diagram

$$
\begin{array}{cccc}
X & \stackrel{\bar{f}}{\rightarrow} & Y & \\
\bar{\omega}_{X} \uparrow & & \downarrow \bar{\omega}_{Y} & |\bar{S}(\bar{f})|=f_{\#}, \\
|\bar{S}(X)| & \stackrel{f_{\#}}{\rightarrow} & |\bar{S}(Y)| &
\end{array}
$$

$\bar{\pi}_{*}\left(\bar{\omega}_{X}\right), \bar{\pi}_{*}\left(\bar{\omega}_{Y}\right)$ being isomorphisms. We have $\bar{\pi}_{*}(|\bar{S}(X)|) \approx \pi_{*}(|\bar{S}(X)|)$ (resp. for $Y$ ) so that together with $\pi_{*}\left(f_{\#}\right)$ also $\bar{\pi}_{*}\left(f_{\#}\right)$ and therefore $\bar{\pi}_{*}(\bar{f})$ is an isomorphism. This proves the second part. Assume $X, Y$ being $s$-connected; then $|\bar{S}(X)|,|\bar{S}(Y)|$ are connected. Suppose that $\bar{\pi}_{*}(\bar{f})$ is an isomorphism; then $\pi_{*}\left(f_{\#}\right)$ is an isomorphism and the conclusion follows from the classical Whitehead theorem.

This enables us to define:

$$
\tilde{\mathfrak{S}}_{*}(X)=\mathfrak{S}_{*}(|\bar{S}(X)|)
$$


and for any $\bar{f} \in \bar{K}(X, Y)$

$$
\tilde{\mathfrak{S}}_{*}(\bar{f})=\mathfrak{H}_{*}(|\bar{S}(\bar{f})|) .
$$

We get a natural transformation $\tilde{\boldsymbol{\sigma}}: \tilde{\mathfrak{S}}_{*}() \rightarrow \tilde{\mathfrak{S}}_{*+1}(\Sigma)$ as follows: We have a natural mapping

$$
\lambda: S^{1} \wedge|\bar{S}(X)| \rightarrow\left|\bar{S}\left(S^{1} \wedge X\right)\right|=|\bar{S}(\Sigma X)|
$$

for any space $X$ (cf. Theorem 4.1 in [4] and Theorem 2.1 below). So we can define

$$
\tilde{\boldsymbol{\sigma}}: \tilde{\mathfrak{S}}_{*}(X) \rightarrow \tilde{\mathscr{H}}_{*+1}(\Sigma X)
$$

by composing $\tilde{\sigma}$ with $\mathfrak{S}_{*+1}(\lambda)$. Lemma 1.4 implies:

1.5. LEMMA. Let $\mathfrak{S}_{*}: \underline{P}_{1 h} \rightarrow \mathrm{Ab}^{\mathrm{Z}}$ be a given homology theory, then $\mathfrak{H}_{*}$ : $\bar{K}_{h} \rightarrow \mathrm{Ab}^{\mathrm{Z}}$ is a homology theory (i.e. one, satisfying the Whitehead axiom $\underline{\mathrm{W}})$. Moreover the natural transformation $\bar{\omega}:|\bar{S}()| \rightarrow 1_{\underline{\underline{K}}}$ induces a natural isomorphism

$$
\tilde{\mathfrak{S}}_{*} \mid \underline{P}_{1} \approx \mathfrak{S}_{*}
$$

We summarize:

1.6. THEOREM. To each homology theory $\mathfrak{S}_{*}: \underline{P}_{1 h} \rightarrow \mathrm{Ab}^{\mathrm{Z}}$ there exists a homology theory $\tilde{\mathfrak{S}}_{*}: \underline{\bar{K}}_{h} \rightarrow \underline{\mathrm{Ab}}$ and a natural isomorphism $\tilde{\mathfrak{S}}_{*} \mid \underline{P}_{1} \approx \mathfrak{S}_{*}$.

The importance of the concept of compact support is well-known from classical algebraic topology. In shape theory we have to define this notion in the following way:

1.7. Definition. A homology theory $\mathscr{S}_{*}: \underline{\bar{K}}_{h} \rightarrow \underline{\mathrm{Ab}}{ }^{\mathrm{Z}}$ is defined to have compact support whenever the following holds:

(C) (a) To any $\zeta \in \mathfrak{S}_{n}(X), X \in \underline{K}$ there exists a compact metric $K$, a $\bar{f} \in \underline{\bar{K}}(K, X)$ and a $\zeta^{\prime} \in \mathscr{S}_{n}(K)$ such that

$$
\mathfrak{S}_{n}(\bar{f})\left(\zeta^{\prime}\right)=\bar{f}_{*}\left(\zeta^{\prime}\right)=\zeta .
$$

(b) Let $\zeta^{\prime}, K, \bar{f}$ be as in (a) and assume that $\bar{f}_{*}\left(\zeta^{\prime}\right)=0$. Then there exists a homotopy commutative diagram in $\underline{\bar{K}}$

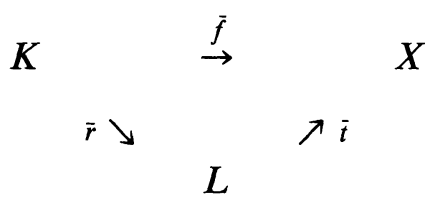

with compact metric $L$, such that $\bar{r}_{*}\left(\zeta^{\prime}\right)=0$. 
On the other hand let $\mathfrak{S}_{*}: \underline{P}_{1 h} \rightarrow \mathrm{Ab}^{\mathrm{Z}}$ be a homology theory, then we say that $\mathscr{S}_{*}$ has compact support, whenever the following holds:

(PC) (a) Let $\zeta \in \mathfrak{S}_{*}(X)$ be any element, then there exists a compact subspace $K \subset X, K \in \underline{P}_{1}$ and a $\zeta^{\prime} \in \mathscr{F}_{*}(K)$ such that $\mathscr{S}_{*}(i)\left(\zeta^{\prime}\right)=\zeta$, $i: K \subset X$ denoting the inclusion.

(b) Let $\zeta^{\prime}, K$, be as in (a) such that now $i_{*}\left(\zeta^{\prime}\right)=\mathscr{F}_{*}(i)\left(\zeta^{\prime}\right)=0$, then there exists a $L \in \underline{P}_{0}, K \subset L \subset X$, such that $\mathfrak{S}_{*}(j)\left(\zeta^{\prime}\right)=0(j: K \subset L$ being the inclusion).

REMARK. The difference between ( $\underline{\mathrm{C}})$ and $(\mathrm{PC})$ is caused by the fact that a continuous mapping $f: K \rightarrow X \in \underline{P}_{1}, \bar{K}$ compact, has always a compact image. This is no longer true for shape mappings: Even for a point $*$ and a shape mapping $\bar{f} \in \underline{\bar{K}}(*, X)$ into a non-compact space $X$, there does in general not exist a compact $K \subset X$ over which $\bar{f}$ factors.

The relation between $(\underline{C})$ and $(\mathrm{PC})$ is embodied in the following:

1.8. Proposition. Let $\mathfrak{S}_{*}: \underline{P}_{1 h} \rightarrow \mathrm{Ab}^{\mathrm{Z}}$ be a homology theory satisfying (PC). Then the extension $\left.\tilde{\mathfrak{S}}_{*}()=\mathfrak{S}_{*}(\mid \overline{S(}) \mid\right): \underline{\bar{K}}_{h} \rightarrow \mathrm{Ab}^{\mathrm{z}}$ of $\mathfrak{S}_{*}$ over $\underline{\bar{K}}_{h}$ satisfies $(\underline{\mathrm{C}})$.

Proof. Let $\zeta \in \mathscr{S}_{*}(|\bar{S}(X)|)$ be a given element, then there exists a finite subcomplex $K \subset|\bar{S}(X)|$ and a $\zeta^{\prime \prime} \in \mathfrak{S}_{*}(K)$ such that $\mathfrak{S}_{*}(i)\left(\zeta^{\prime \prime}\right)=$ $i_{*}\left(\zeta^{\prime \prime}\right)=\zeta$.

Moreover we have the following commutative diagram

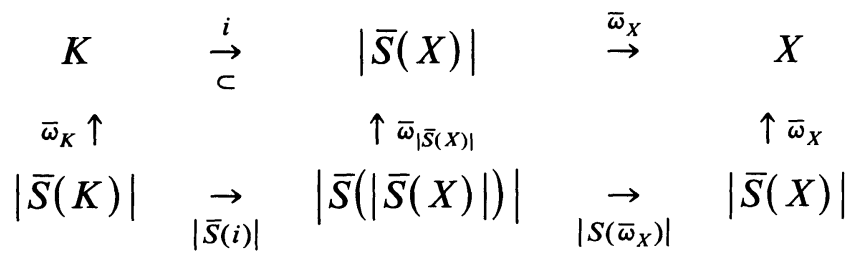

where $\bar{\omega}_{K}$ is a homotopy equivalence in $\underline{\bar{K}}$ between $\mathrm{CW}$ spaces, hence homotopic to a continuous homotopy equivalence $\omega$ in $\underline{K}$ (cf. 7.1). The mapping $\tilde{\mathfrak{S}}_{*}\left(\bar{\omega}_{X}\right)=\mathfrak{S}_{*}\left(\left|\bar{S}\left(\bar{\omega}_{X}\right)\right|\right)$ is an isomorphism (cf. Lemma 1.5). Setting $\zeta^{\prime}=\mathscr{S}_{*}(\omega)^{-1}\left(\zeta^{\prime \prime}\right)$ and $\bar{f}=\bar{\omega}_{X} i$, we conclude

$$
\mathfrak{S}_{*}\left(\left|\bar{S}\left(\bar{\omega}_{X}\right)\right|\right) \mathfrak{S}_{*}(|\bar{S}(\bar{f})|)\left(\zeta^{\prime}\right)=\mathfrak{S}_{*}\left(\left|\bar{S}\left(\bar{\omega}_{X}\right)\right|\right) \mathfrak{S}_{*}(i) \mathfrak{S}_{*}(\omega)\left(\zeta^{\prime}\right)
$$


therefore

$$
\tilde{\mathfrak{S}}_{*}(\bar{f})\left(\zeta^{\prime}\right)=\mathfrak{S}_{*}(i)\left(\zeta^{\prime \prime}\right)=\zeta .
$$

This proves $(\underline{\mathrm{C}})(\mathrm{a})$. The proof of $(\underline{\mathrm{C}})(\mathrm{b})$ is similar.

2. The homology theory $\underline{E}_{*}$. A spectrum $\underline{E}=\left\{E_{n}, \sigma_{n}: E_{n} \rightarrow\right.$ $\left.E_{n+1}, n \in Z\right\}$ is a sequence of based spaces and continuous maps $\sigma_{n}$. A $\mathrm{CW}$ spectrum has the additional property that all $E_{n}$ are $\mathrm{CW}$ complexes and that all $\sigma_{n}$ are supposed to be cellular. The category Spec has these $\mathrm{CW}$ spectra as objects and so-called functions of spectra $f=$ $\left\{f_{n}: E_{n} \rightarrow F_{n}\right\}$ as morphisms, where we assume that the $f_{n}$ are compatible with the corresponding $\sigma_{n}: \Sigma E_{n} \rightarrow E_{n+1}$ resp. $\Sigma F_{n} \rightarrow f_{n+1}$.

The following full subcategory CSpec $\subset$ Spec deserves particular interest: Its objects are those spectra $\left.\overline{E=\{} E_{n}\right\}$, having the property that (1) all $m$-skeletons $\left(E_{n}\right)^{m}$ are for all $m$ and all $n \in \mathbf{Z}$ compact and that (2) there exists a $n_{0}$ such that all $E_{n}, n \geq n_{0}$ are simply connected. We call such a spectrum also a cs-spectrum. It can be verified that an Eilenberg-Mac Lane spectrum $K(G)$ for finitely generated abelian group $G$ has this property.

Let Com $\subset \bar{K}$ be the full subcategory of based compacta, then we have defined in [4] the homology theory

$$
\underline{E}_{n}(X)=\lim _{\vec{k}} \bar{\pi}_{n+k}\left(E_{k} \bar{\wedge} X\right), \quad X \in \text { Com. }
$$

We have that $\bar{E}_{*}: \overline{\mathrm{Com}}_{h} \rightarrow \mathrm{Ab}^{\mathrm{z}}$ is a functor. Moreover there are natural isomorphisms $\sigma=\bar{\sigma}_{n}: \underline{E}_{n} \approx \overline{\bar{E}}_{n+1} \cdot \Sigma$. However the Whitehead axiom (W) is not necessarily fulfilled so $\underline{E}_{*}=\left\{\bar{E}_{n}, \sigma\right\}$ is not always a shape singular homology theory (cf. Definition 1.3).

A space $X \in$ Com is called s-continuum, whenever $\bar{\pi}_{0}(X)=0$.

So the solenoi for example is not $s$-connected, while it can be proved that the double suspension of the solenoid is a $s$-continuum. Let $E$ be a CW complex, $X$ a compactum, then we have a homotopy equivalence

$$
E \wedge|\bar{S}(X)| \simeq|\bar{S}(E)| \wedge|\bar{S}(X)|
$$

and a continuous mapping $\lambda:|\bar{S}(E)| \wedge|\bar{S}(X)| \rightarrow|\bar{S}(E \bar{\wedge} X)|$. As a consequence of Theorem 4.1 in [4] we have:

2.1. THEOREM. Let $X$ be a s-continuum, $E$ a simply connected $C W$ complex of finite type (i.e. all $m$-skeletons are compact), then $\lambda$ induces $a$ natural homotopy equivalence

$$
|\bar{S}(E \bar{\wedge} X)| \stackrel{\simeq}{\leftarrow}|\bar{S}(E)| \wedge|\bar{S}(X)| \simeq E \wedge|\bar{S}(X)|,
$$


rendering the following diagram homotopy commutative:

$$
\begin{array}{rlc}
|\bar{S}(E)| \wedge|\bar{S}(X)| & \stackrel{\bar{\omega}_{E} \wedge 1}{\rightarrow} & E \wedge|\bar{S}(X)| \\
\downarrow \Downarrow & & \downarrow 1 \wedge \bar{\omega}_{X} \\
|\bar{S}(E \bar{\wedge} X)| & \underset{\bar{\omega}_{E \wedge X}}{\rightarrow} & E \bar{\wedge} X
\end{array}
$$

We define $\underline{E}_{n}(X)=\lim _{\rightarrow} \pi_{n+k}\left(E_{k} \wedge X\right)$ (all mappings and homotopies being continuous). As a consequence we have:

2.2. TheOREM. (CF. [4] Theorem 4.2.) Let the s-continuum $X$ and the spectrum $\underline{E} \in \mathrm{CSpec}$ be given, then there exists a natural isomorphism

$$
\bar{E}_{*}(X) \approx \underline{E}_{*}(|\bar{S}(X)|) \text {. }
$$

Proof. We have the following series of isomorphisms

$$
\begin{aligned}
\underline{E}_{n}(X) & =\underset{\vec{k}}{\lim } \bar{\pi}_{n+k}\left(E_{k} \pi X\right) \approx \underset{\vec{k}}{\lim } \pi_{n+k}\left(\left|\bar{S}\left(E_{k} \bar{\wedge} X\right)\right|\right) \\
& \approx \lim _{\vec{k}} \pi_{n+k}\left(E_{k} \wedge|\bar{S}(X)|\right)=\underline{E}_{n}(|\bar{S}(X)|),
\end{aligned}
$$

which are clearly compatible with the natural transformations $\sigma$.

In accordance with $\S 1(1),(2)$ we denote the homology theory

$$
\underline{E}_{*}(|\bar{S}()|): \underline{\bar{K}}_{h} \rightarrow \underline{\mathrm{Ab}}^{\mathbf{Z}}
$$

by $\underline{E}_{*}$.

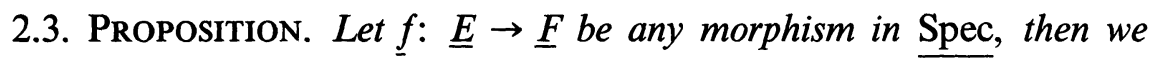
have (1) an induced natural transformation

$$
\underline{f_{\#}}: \bar{E}_{*} \rightarrow \bar{F}_{*}
$$

and (2) a natural transformation

$$
\underline{\tilde{f}}_{\#}: \underline{\tilde{E}}_{*} \rightarrow \underline{\tilde{F}}_{*} .
$$

Proof. This is in both cases an immediate consequence of the definitions of $\underline{E}_{*}$ resp. of $\underline{E}_{*}$ : For example in the first case we have

$$
E_{k} \bar{\wedge} \underset{f_{k} \overrightarrow{\pi 1}_{X}}{\rightarrow} F_{k} \bar{\wedge} X
$$


which is well-defined (cf. [4] §2). This provides us easily with the required $\underline{f_{\#}}$.

2.4. Proposition. Let $\underline{E} \in \mathrm{Spec}, X \in \underline{K}$ and $\zeta \in \tilde{E}_{n}(X)$ be given, then there exists (1) a cs-spectrum $\underline{E}^{\prime}$ (2) a $\underline{\underline{S}} \in \operatorname{Spec}\left(\underline{E}^{\prime}, \underline{E}\right)$ and (3) a $\zeta^{\prime} \in \tilde{E}_{n}^{\prime}(X)$ such that $\tilde{\varphi}_{\#}\left(\zeta^{\prime}\right)=\zeta$. Moreover assume $\zeta^{\prime}, \underline{E}^{\prime}, \underline{\varphi}$ be given such that $\underline{\tilde{\varphi}}_{\#}\left(\zeta^{\prime}\right)=0$, then there exists a $\underline{E}^{\prime \prime} \in \mathrm{CSpec}$ as well as a factorization

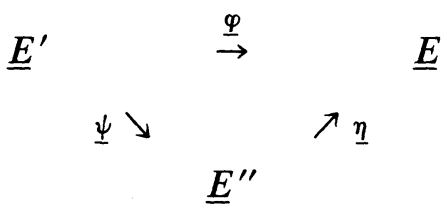

such that $\tilde{\Psi}_{\#}\left(\zeta^{\prime}\right)=0$.

Proof. We abbreviate $|\bar{S}(X)|$ by $Y$ and call a $\underline{E} \in$ Spec simple whenever there exists a $n_{0} \in Z$ such that all $E_{n}, n \geq n_{0} \overline{\text { are }}$ simply connected. Let us in a first step deal with a simple spectrum $\underline{E}$. We construct $\underline{E}^{\prime}, \varphi$ in the following way:

Let $\left(f: S^{\bar{n}+k} \rightarrow E_{k} \wedge Y\right.$ ) be a representative of $\zeta$ (observe that $Y$ is a CW space!). Then there exists a compact $E_{k}^{\prime} \subset E_{k}$ such that $f$ factors over $E_{k}^{\prime} \wedge Y$. By eventually suspending $f$ we obtain a $E_{k}^{\prime}$ which is simply connected. Now we set $E_{l}^{\prime}=*$ for $l<k$. In order to establish $E_{k+1}^{\prime}$ we take $\sigma_{k}\left(\Sigma E_{k}^{\prime}\right) \subset E_{k+1}$ and kill the fundamental group of this space in $E_{k+1}$ (which is by assumption simply connected). This provides us with a $E_{k+1}^{\prime} \subset E_{k+1}$ and a $\sigma_{k}^{\prime}: \Sigma E_{k}^{\prime} \rightarrow E_{k+1}^{\prime}$. Proceeding inductively we obtain a $\underline{\varphi}: \underline{E}^{\prime} \rightarrow \underline{E}$ such that $\underline{E}^{\prime} \in \mathrm{CSpec}$ and a $\zeta^{\prime} \in \underline{E}_{*}(Y)$ with $\underline{\varphi}_{\#}\left(\zeta^{\prime}\right)=\zeta$. The second statement is proved similarly.

This completes the proof of the assertion for simple spectra. In order to get rid of this last assumption, we construct to any $\underline{E} \in \mathrm{Spec}$ the following spectrum $\underline{\hat{E}}=\left\{\hat{E}_{n}\right\}$ :

$$
\begin{aligned}
\hat{E}_{n} & =\Sigma^{2} E_{n-2}, \\
\Sigma^{2} \sigma_{n-2} & =\hat{\sigma}_{n}: \Sigma \hat{E}_{n}=\Sigma^{3} E_{n-2} \rightarrow \Sigma^{2} \hat{E}_{n-1}=\hat{E}_{n+1} .
\end{aligned}
$$

This spectrum is clearly simple. There exists a morphism $\tau_{\underline{E}}=\tau: \underline{\hat{E}} \rightarrow \underline{E}$, $\tau_{n}=\sigma_{n-1} \cdot \Sigma \sigma_{n-2}: \quad \hat{E}_{n}=\Sigma^{2} E_{n-2} \rightarrow E_{n}, \quad \tau \in \operatorname{Spec}(\underline{\hat{E}}, \underline{E})$. On $\underline{P}_{1}$ this transformation clearly induces a natural isomorphism

$$
\tau_{*}: \hat{E}_{n}() \approx \underline{E}_{n}() \text {, }
$$

hence a natural isomorphism

$$
\tau_{*}: \underline{\hat{E}}_{n}() \approx \underline{\tilde{E}}_{n}() \text {. }
$$


Now we can apply the previous construction of $\underline{E}^{\prime}$ to $\underline{\hat{E}}$ (instead of $\underline{E}$ ) thereby completing the proof of 2.4 .

REMARK. There exist a $s$-continuum $X$ and a not finitely generated abelian group $G$ such that

$$
\overline{K(G)}_{n}(X) \neq{\overline{K(G)_{n}}}_{n}(X) .
$$

(Cf. remark following 6.5.)

In particular this confirms that 2.4 does not hold for $\overline{K(G)}_{*}$ (even not for $s$-connected compacta).

2.5. TheOREM. The homology theory $\underline{E}_{*}: \underline{K}_{h} \rightarrow \mathrm{Ab}^{\mathrm{Z}}$ has compact support (i.e. satisfies $\underline{C}$ ) in Definition 1.7.

Proof. The fact that $\underline{E}_{*}$ is a homology theory follows from Lemma 1.5. Because we have a natural isomorphism $\underline{E}_{*}() \approx \underline{E}_{*}()$ on $\underline{P}_{1}$ and since $\underline{E}_{*}$ on $\underline{P}_{1}$ has compact support, the assertion follows from 1.8 .

REMARK. In general we cannot expect that

$$
\sigma: \underline{E}_{n}(X) \rightarrow \underline{E}_{n+1}(\Sigma X)
$$

is becoming an isomorphism, unless $X$ is a shape simply connected compactum (i.e. one has $\bar{\pi}_{i}(X)=0$ for $\left.i=0,1\right)$. In the latter case we have due to Theorem 4.1 in [4] a homotopy equivalence

$$
\left|\bar{S}\left(S^{1} \wedge X\right)\right| \simeq S^{1} \wedge|\bar{S}(X)|
$$

furnishing an isomorphism

$$
\tilde{E}_{n}(X)=\underline{E}_{n}(|\bar{S}(X)|) \approx \underline{E}_{n+1}\left(S^{1} \wedge|\bar{S}(X)|\right)=\underline{\tilde{E}}_{n+1}(\Sigma X) .
$$

3. The uniqueness theorem. Let $\mathfrak{S}_{*}, \mathfrak{S}^{\prime}{ }_{*}: \underline{\bar{K}}_{h} \rightarrow \mathrm{Ab}^{\mathrm{Z}}$ be two homology theories (i.e. those satisfying $(\underline{W})$ ). Suppose we have a natural transformation of homology theories $\alpha: \mathfrak{S}_{*}\left|\underline{P}_{1 h} \rightarrow \mathfrak{S}^{\prime}{ }^{\prime}\right| \underline{P}_{1 h}$.

\footnotetext{
$\bar{K}_{h}$.

3.1. ThEOREM. There exists a unique extension $\tilde{\alpha}: \mathfrak{S}_{*} \rightarrow \mathfrak{S}^{\prime}{ }^{\prime}$ of $\alpha$ over
}

Proof. The natural transformation $\bar{\omega}_{X}:|\bar{S}(X)| \rightarrow X$ is a weak homotopy equivalence in $\underline{K}_{h}$, hence we are allowed to define

$$
\tilde{\boldsymbol{\alpha}}_{X}: \mathfrak{S}_{*}(X) \rightarrow \mathfrak{S}^{\prime}{ }_{*}(X)
$$

by

$$
\tilde{\alpha}_{X}=\mathfrak{S}^{\prime}{ }_{*}\left(\bar{\omega}_{X}\right) \alpha_{|\bar{S}(X)|} \mathfrak{S}_{*}\left(\bar{\omega}_{X}\right)^{-1}
$$


The following assertions are all more or less immediate.

(1) $\tilde{\alpha}$ is natural with respect to $X$.

Proof. This is obvious because $\bar{\omega}:|\bar{S}()| \rightarrow 1_{\underline{\underline{K}}}$ as well as $\alpha$ are natural.

(2) $\tilde{\alpha}$ is a homomorphism. This is trivial.

(3) $\tilde{\alpha} \mid \underline{P}_{1 h}=\alpha$.

Proof. Let $X \in \underline{P}_{1}$ be a $\mathrm{CW}$ space, then we have a commutative diagram

$$
\begin{array}{ccc}
\mathfrak{S}_{*}(X) & \stackrel{\mathfrak{E}_{*}\left(\bar{\omega}_{X}\right)}{\leftarrow} & \mathfrak{S}_{*}(|\bar{S}(X)|) \\
\alpha_{X} \downarrow & & \downarrow \alpha_{|\bar{S}(X)|} \\
\mathfrak{S E}_{*}^{\prime}(X) & \stackrel{\mathfrak{Q}_{*}^{\prime}\left(\bar{\omega}_{X}\right)}{\leftarrow} & \mathfrak{S E}_{*}^{\prime}(|S(X)|)
\end{array}
$$

and therefore

$$
\tilde{\alpha}_{X}={\mathfrak{S}^{\prime}}_{*}\left(\bar{\omega}_{X}\right) \alpha_{|\bar{S}(X)|} \mathfrak{S}_{*}\left(\bar{\omega}_{X}\right)^{-1}=\alpha_{X} .
$$

(4) $\tilde{\alpha}$ is compatible with the bonding maps $\sigma, \sigma^{\prime}$.

Proof. This follows immediately because $\alpha$ is supposed to have this property on the category $P_{1}$.

(5) Let $\beta: \mathfrak{S}_{*} \rightarrow \mathscr{S}^{\prime}{ }^{\prime}$ be any natural transformation extending $\alpha$, then we have $\beta=\tilde{\alpha}$.

Proof. This is trivial in view of (2) (now for any $X \in \underline{K}$ and after replacing $\alpha_{X}$ by $\beta_{X}$ ).

This completes the proof of Theorem 3.1.

We have the following corollaries:

3.2. Corollary. Let $\alpha: \mathfrak{S}_{*}\left|\underline{P}_{1 h} \approx \mathfrak{S}^{\prime}{ }^{\prime}\right| \underline{P}_{1 h}: \underline{P}_{1 h} \rightarrow \mathrm{Ab}^{\mathrm{Z}}$ be an isomorphism of homology theories, then there exists a unique isomorphism $\tilde{\alpha}$ : $\mathfrak{S}_{*} \approx \mathfrak{S}^{\prime}{ }^{\prime}$ on $\underline{K}_{h}$, extending $\alpha$. 
3.3. CoRollaRY. Let $\mathfrak{S}_{*}: \underline{K}_{h} \rightarrow \mathrm{Ab}^{\mathrm{Z}}$ be any homology theory and $\alpha$ : $\mathfrak{S}_{*} \approx \underline{E}_{*}$ an isomorphism of homology theories on the category $\underline{P}_{1 h}$. Then there exists a unique extension $\tilde{\alpha}: \mathfrak{S}_{*} \approx \underline{E}_{*}$ of $\alpha$ to an isomorphism on the category $\underline{K}_{h}$. Furthermore $\mathfrak{S}_{*}$ is of compact support (Definition 1.7).

Proof. The first part follows simply by applying Theorem 3.1 to $\mathfrak{S}^{\prime}{ }_{*}=\tilde{E}_{*}$. The second part follows from the first because 2.5 makes sure that $\underline{E}_{*} \approx \mathscr{S}_{*}$ is of compact support.

4. $\bar{E}_{*}$ and the clusteraxiom. In [12] J. Milnor introduced the clusteraxiom (or strong wedge axiom) for a homology theory $H_{*}$ defined on the category of compact metric spaces (with base-points).

(C1) Let $\left(X_{i}, x_{0 l}\right)=X_{i}, i=1,2, \ldots$ be a sequence of compact metric spaces and

$$
\stackrel{\infty}{\mathrm{C} l} X_{\imath}=\underset{m}{\lim }\left(X_{1} \vee \cdots \vee X_{m}\right)
$$

the cluster (or strong wedge), then the natural homomorphism

$$
H_{*}\left(\stackrel{\infty}{\mathrm{C} l} X_{i}\right) \rightarrow \prod_{i=1}^{\infty} H_{*}\left(X_{\imath}\right)
$$

becomes an isomorphism.

$\mathrm{He}$ was able to show that the Steenrod-Sitnikov homology $H_{*}^{S}(X, Y ; G)$ groups with arbitrary coefficient group $G$ can be characterized (now in the category of compact metric pairs) by the ordinary Eilenberg-Steenrod axioms (with a strong excision axiom $H_{*}^{S}(X, Y ; G) \approx$ $\left.H_{*}^{S}(X / Y, * ; G)\right)$ together with the clusteraxiom.

In this section we are going to find out under what circumstances $\bar{E}_{*} h:$ Com $\rightarrow \mathrm{Ab}^{\mathrm{z}}$ for a given $\mathrm{CW}$ spectrum $\underline{E}$ fulfills the cluster axiom.

We formulate the following property of a $\mathrm{CW}$-spectrum:

(S) There exists a $p \in \mathbf{Z}$ having the following property:

The mapping $\sigma: \Sigma E_{i} \rightarrow E_{i+1}$ induces an isomorphism of the $(2 i+p)$-skeleton for all $i$.

We are able to prove:

4.1. Proposition. Let $\underline{E} \in \mathrm{Spec}$ be such that $(\underline{\mathrm{S}})$ is satisfied, then the cluster axiom $(\underline{\mathrm{Cl}})$ holds for $\underline{\bar{E}}_{*}$.

Proof. Set $X=\mathrm{Cl}_{i=1}^{\infty} X_{i}$ and let

$$
p_{n}^{n+1}: \bigvee_{i=1}^{n+1} X_{i} \rightarrow \bigvee_{i=1}^{n} X_{i}
$$


resp.

$$
q_{n}: \stackrel{\infty}{\mathrm{C}} X_{i} \rightarrow X_{n}
$$

be the projections. We have the natural homomorphism

$$
\varphi: \underline{E}_{*}(X) \rightarrow \prod_{i=1}^{\infty} \bar{E}_{*}\left(X_{i}\right)
$$

defined by

$$
\varphi(\zeta)=\left\{q_{i *}(\zeta)\right\}
$$

In order to construct an inverse to $\varphi$, let $\left\{\zeta_{i}\right\} \in \prod_{i=1}^{\infty} \bar{E}_{n}\left(X_{i}\right)$ be any element in the direct product and $\zeta_{i} \ni \bar{f}_{i}: S^{n+1(i)} \rightarrow E_{1(i)} \wedge X_{i}$ be representatives. We fix a continuous $g_{i}: X_{i} \rightarrow P_{i} \in \underline{P}$ for each $i$ and define

$$
f_{i}=\bar{f}_{i}\left(g_{i}\right): S^{n+l(i)} \rightarrow E_{l(i)} \wedge P_{i} .
$$

We have to distinguish two cases:

1. $l(i)>n-p+2$ : We put $q=p-2$ and observe that $f_{i}$ factors over $\left(E_{l(i)} \wedge P_{i}\right)^{n+l(i)} \subset\left(E_{l(i)} \wedge P_{i}\right)^{2 l(i)+q}$. However for cellular reasons we have:

$$
\left(E_{l(i)} \wedge P_{i}\right)^{2 l(i)+q} \subset\left(E_{l(i)}\right)^{2 l(i)+q} \wedge P_{i}
$$

and, by our assumption ( $(\underline{\mathbf{S}})$, an isomorphism

$$
\left(E_{l(i)}\right)^{2 l(i)+q} \wedge P_{i} \approx\left(\Sigma E_{l(i)-1}\right)^{2 l(i)+q} \wedge P_{i} .
$$

Hence $f_{i}$ turns out to be stably homotopic to a

$$
f_{i}^{\prime}: S^{n+1(i)} \rightarrow \Sigma\left(E_{l(i)-1} \wedge P_{i}\right)
$$

because we have

$$
\left(\Sigma E_{l(i)-1}\right)^{k} \approx \Sigma\left(E_{l(i)-1}\right)^{k-1}
$$

for all $k \in N$, this procedure can be iterated until we reach a

$$
f_{i}^{\prime \prime}: S^{n+l(i)} \rightarrow \Sigma^{l(i)-(n-q)}\left(E_{n-q} \wedge P_{i}\right) .
$$

Putting $k=l(i)-n+1$ we have $n+l(i)=2 n-q+k$ and therefore we find

$$
f_{i}^{\prime \prime} \in \pi_{2 n-1+k}\left(\Sigma^{k}\left(E_{n-q} \wedge P_{i}\right)\right) .
$$

The stability theorem ensures the existence of an index $m$ such that the suspension homomorphism

$$
\Sigma_{*}: \pi_{2 n-1+k}\left(\Sigma^{k}\left(E_{n-q} \wedge P_{i}\right)\right) \rightarrow \pi_{2 n-1+k+1}\left(\Sigma^{k+1}\left(E_{n-q} \wedge P_{i}\right)\right)
$$


becomes an isomorphism for $k \geq m$. Therefore the number

$$
l=n-q+m
$$

has the property that to each $f_{i}$ there always exists a representative $\hat{f}_{i}$ : $S^{n+l} \rightarrow E_{l} \wedge P_{i}$. Observe that $l$ is independent of $i, l(i)$ and $g_{i}$. As a result we can henceforth assume without loss of generality that $\bar{f}_{i} \in \zeta_{i}$ is of the form:

$$
\bar{f}_{i} \in \underline{K}_{h}\left(S^{n+l}, E_{l} \bar{\wedge} X_{i}\right), \quad n+l \geq 1,
$$

with a universal $l$ (not depending upon $i$ ).

The second case:

2. $l(i) \leq n-p+2$ : can be settled by eventually suspending until we reach the same universal level $l$ for all $i$.

Now it turns out to be rather simple to compose all these $\bar{f}_{i}$, yielding a $\psi\left\{\bar{f}_{i}\right\}: S^{n+l} \rightarrow E_{l} \bar{\wedge} X$.

To this end we must apply the explicit construction of a shape mapping, laid down, for example, in [4] appendix: Let $g: X \rightarrow P \in \underline{P}$ be any object in $\underline{P}_{X}$, then we factorize over a suitable

$$
g^{m}: X \rightarrow \bigvee_{j=1}^{m} P_{j}, \quad g^{m}=\left(\bigvee_{j=1}^{m} g_{i}\right) p^{m}
$$

where

$$
p^{m}: \stackrel{C}{i=1}_{i}^{\infty} X_{i} \rightarrow \bigvee_{i=1}^{m} X_{i}
$$

denotes the projection and $g_{j}: X_{j} \rightarrow P_{j}$ is a given object in $\underline{P}_{X_{j}}$. Hence we merely have to evaluate $\psi\left\{\bar{f}_{i}\right\}$ on mappings of the form $g^{m}$. This can obviously be accomplished by

$$
\psi\left\{\bar{f}_{i}\right\}\left(g^{m}\right)=\left(f_{1} \vee \cdots \vee f_{m}\right) \kappa
$$

where $\kappa: S^{n+l} \rightarrow S^{n+l} \vee \cdots \vee S^{n+l}$ denotes the $m$-fold comultiplication and where we put: $f_{i}=\bar{f}_{i}\left(g_{i}\right)$ as before. The verification of the fact that (1) establishes a

$$
\psi\left\{\bar{f}_{i}\right\}: S^{n+l} \rightarrow E_{l} \bar{\wedge} X
$$

is in view of the techniques developed in [4] appendix merely routine. This gives rise to a mapping $\psi: \prod_{i=1}^{\infty} \underline{E}_{n}\left(X_{i}\right) \rightarrow \underline{E}_{n}(X)$ having the property

$$
\varphi \psi=1 \text {. }
$$


We still have to verify that $\varphi$ is also a monomorphism. To this end let

$$
\left(\bar{f}: S^{n+r} \rightarrow E_{r} \bar{\wedge} X\right) \in \zeta \in \underline{E}_{n}(X)
$$

be given and consider

$$
\bar{f}_{i}=\left(1_{E_{r}} \bar{\wedge} q_{i}\right) \bar{f}: S^{n+r} \rightarrow E_{r} \bar{\wedge} X_{i} .
$$

Assume the existence of indexes $l(i) \geq r$ (eventually depending upon $i$ ) such that the corresponding representatives $\bar{f}_{i}^{\prime}: S^{n+l(i)} \rightarrow E_{l(i)} \bar{\wedge} X_{i}$ are null-homotopic.

The same kind of reasoning as above provides us with a universal index $l$ and representatives

$$
\bar{f}_{i}^{\prime \prime}: S^{n+l} \rightarrow E_{l} \bar{\wedge} X_{i}
$$

such that $\bar{f}_{i}^{\prime \prime} \simeq 0$. Now these homotopies can be composed to a unified homotopy of the original $\bar{f}$ to the constant map (in the same way in which we constructed $\psi\left\{\bar{f}_{i}\right\}$ before). This assures us that $\varphi$ is a monomorphism and completes the proof of the proposition.

There are two basic applications of this result, which we are going to develop:

Recall that a spectrum $\underline{E}$ is called connective, whenever

(1) $E$ is an $\Omega$-spectrum (i.e. one has $E_{i} \simeq \Omega E_{i+1}$ ) and

(2) $E_{i}$ is $(i-1)$ connected (a condition which is of course meaningless for $i<0$ ).

We have the following general assertion:

4.2. LEMMA. Let $\underline{E}$ be connective, then there exists a spectrum $\underline{E}^{\prime}=$ $\left\{E_{i}^{\prime}\right\} \in \operatorname{Spec}$ such that $(\underline{\mathbf{S}})$ holds as well as a function of spectra $f: \underline{E} \rightarrow \underline{E}^{\prime}$ with $f_{n} \overline{\text { being }}$ a homotopy equivalence for all $n$. In particular $\underline{f}$ induces $a$ natural isomorphism $\underline{\underline{E}}_{*} \approx \underline{E}_{*}^{\prime}$.

Proof. This is a quite elementary fact which can be proved inductively: Setting $E_{i}^{\prime}=E_{i}$ for $i \leq 0$, we assume that $E_{m}^{\prime}$ and $f_{m}$ have already been constructed for $m \leq n$. According to the stability theorem we obtain a suspension isomorphism

$$
\pi_{i+1}\left(E_{n+1}\right) \approx \pi_{i}\left(E_{n}^{\prime}\right) \approx \pi_{i+1}\left(E_{n}^{\prime}\right)
$$

for $i \leq 2(n-1)$.

This isomorphism is easily recognized to be induced by $\tau_{n}: \Sigma E_{n}^{2} \rightarrow$ $E_{n+1}$ (with $\tau_{n}$ being defined by $\sigma_{n}=\left(\Sigma f_{n}\right) \tau_{n}$ ): Let $\sigma_{n}^{\prime}$ resp. $p$ be the adjoints to $\sigma_{n}$ resp. the identity 1: $\Sigma E_{n}^{\prime} \rightarrow \Sigma E_{n}^{\prime}$, then we have

$$
\left(\Omega \tau_{n}\right) p=\tau_{n}^{\prime} \text {. }
$$


As a result we have a commutative diagram

$$
\begin{array}{lll}
\pi_{i+1}\left(E_{n+1}\right) & \approx & \pi_{i}\left(\Omega E_{n+1}\right) \\
\pi_{n+1} \uparrow & \uparrow \Omega \sigma_{n \#} \\
\pi_{i+1}\left(\Sigma E_{n}^{\prime}\right) & \approx & \pi_{i}\left(\Omega \Sigma E_{n}^{\prime}\right)
\end{array} \overbrace{p_{\text {\# }}}^{\sigma_{n \#}^{\prime}} \pi_{i}\left(E_{n}^{\prime}\right)
$$

Since all induced homomorphisms on the right-hand side are isomorphism (for $i \leq 2(n-1)$ ), we conclude that $\sigma_{n \#}$ is also an isomorphism.

In defining $E_{n+1}^{\prime}$, we set $\left(E_{n+1}^{\prime}\right)^{2 n-3}=\left(\Sigma E_{n}^{\prime}\right)^{2 n-3}$ and recall that $\tau_{n}$ : $\Sigma E_{n}^{\prime} \rightarrow E_{n+1}$ induces an isomorphism of the homotopy groups $\pi_{l}$ for $i \leq 2 n-3$. We attach $2 n-1$-cells to $\Sigma E_{n}^{\prime}$ killing the kernel of $\pi_{2 n-2}\left(\tau_{n}\right)$ and $2 n-2$-cells representing the cokernel of $\pi_{2 n-2}\left(\tau_{n}\right)$. As a result we find a new space $X_{1} \supset \Sigma E_{n}^{\prime}$ and a continuous $f_{1}: X \rightarrow E_{n+1}$, extending $\tau_{n}$ such that $\pi_{i}\left(f_{1}\right)=$ isomorphism for $i \leq 2 n-2$. Proceeding inductively, we find $f_{2}, X_{2}, \ldots$ and finally a CW-complex $X \supset \Sigma E_{n}^{\prime}$ as well as a continuous $f: X \rightarrow E_{n}^{\prime}$ extending $\sigma_{n}$ such that $\pi_{t}(f)$ becomes an isomorphism for all $i$. We define $X=E_{n+1}^{\prime}$ and conclude that $\left(E_{n+1}^{\prime}\right)^{2 n-3}=$ $\left(\Sigma E_{n}^{\prime}\right)^{2 n-3}$, and that there exists a homotopy equivalence $f_{n+1}: E_{n+1}^{\prime} \simeq$ $E_{n+1}$ which is compatible with the resp. bonding maps. $\Sigma E_{n}^{\prime} \stackrel{\sigma_{n}^{\prime}}{\rightarrow} E_{n+1}^{\prime}$ (being the inclusion $\Sigma E_{n}^{\prime} \stackrel{\subset}{\rightarrow} X=E_{n+1}^{\prime}$ ).

As a corollary we obtain our first application:

4.3. Proposition. Let $G$ be any abelian group, then for $\overline{K(G)}_{*}$ the clusteraxiom holds.

Proof. Because $K(G)$, the Eilenberg-Mac Lane spectrum is connective, Lemma 4.2 and finally Proposition 4.1 applies.

Our second application deals with complex $K$-theory $\mathrm{BU}_{*}$ which is determined by the spectrum $\underline{\mathrm{BU}}=\left\{E_{n}\right\}$ with

$$
E_{2 n}=\overline{\mathbf{Z} \times B U,} \quad E_{2 n+1}=U .
$$

We obtain a connective spectrum $b u=\underline{L}=\left\{L_{n}\right\}$ by killing the first ( $i-1)$ buttom homotopy groups of $\bar{E}_{i}$ in a well-known way (e.g. by taking the fibers of certain Postnikov-decompositions of $E_{i}$ ).

Consider the category $\mathrm{Com}^{f}$ of all finite dimensional compact metric spaces, then we have:

(1) An isomorphism

$$
\mathrm{BU}^{n}(X) \approx \underline{L}^{n}(X)
$$

for all CW-spaces $X$ and $n<0$. 
(2) Let $X \in \operatorname{Com}^{f}$ be embedded in some $S^{n+1}$, then we have an isomorphism ([4] Theorem 7.1)

$$
\underline{E}^{n-k}\left(S^{n+1} \backslash X\right) \approx \underline{E}_{k}(X) .
$$

(3) $\overline{\mathrm{BU}}_{*}$ is periodic of period 2, i.e.

$$
\overline{\mathrm{BU}}_{n}(X) \approx \overline{\mathrm{BU}}_{n+2}(X), \quad n \in \mathbf{Z} .
$$

The first statement follows immediately by definition. The second one is simply Alexander duality. The third assertion can be achieved in the same way as in the classical case as an immediate consequence of Bott-periodicity.

4.4. Proposition. On the category $\mathrm{Com}^{f}$ the homology theory $\overline{\mathrm{BU}}_{*}$ fulfills a clusteraxiom.

Proof. Let $X=\mathrm{Cl}_{i=1}^{\infty} X_{i}, X \in \mathrm{Com}^{f}$ be embedded in some $S^{N}$. In establishing the isomorphism

$$
\overline{\mathrm{BU}}_{n}(X) \approx \prod_{i=1}^{\infty} \overline{\mathrm{BU}}_{n}\left(X_{i}\right)
$$

we can in view of (3) without loss of generality assume that $N-n<0$. Under these circumstances we obtain

$$
\underline{\mathrm{BU}}_{n}(X) \approx \underline{\mathrm{BU}}^{N-n}\left(S^{N} \backslash X\right) \approx \underline{L}^{N-n}\left(S^{N} \backslash X\right) \approx \underline{L}_{n}(X) .
$$

Application of Lemma 4.1, 4.2 yields the clusteraxiom for $\bar{L}_{n}(X)$. However the isomorphism (4) is compatible with the inclusions $X_{i} \rightarrow \mathrm{Cl}_{i=1}^{\infty} X_{i}$. On the other hand the desired isomorphism (3) can also be regarded as being induced by these inclusions (rather than by the projections $\left.\mathrm{Cl}_{i=1}^{\infty} X_{i} \rightarrow X_{i}\right)$. This completes the proof of the assertion.

EXAMPLeS. (1) Every suspension spectrum $\underline{E}=\left\{\Sigma^{n} E \mid E \in \underline{P}_{1}\right\}$ has property $(\underline{\mathbf{S}})$.

(2) The same kind of argument which leads to Proposition 4.4 can of course be applied to $\overline{\mathrm{BO}}_{*}$, the homology theory of real $K$-theory.

(3) Let $\underline{E}=\left\{E_{n}\right\}$ be a spectrum having the following property:

(a) $\sigma: \Sigma E_{n} \rightarrow E_{n+1}$ is cellular embedding

(b) $E_{n+1}=S^{n+1} \vee \Sigma E_{n}$.

It is a rather trivial task, to construct a spectrum of this kind. Put $X_{i}=S^{0}=\left\{x_{i}, *\right\}$, then we have

$$
E_{k} \wedge X_{i}=E_{k} \wedge X_{i}=E_{k}
$$


Let $\zeta_{i} \in \underline{E}_{0}\left(X_{i}\right)$ be defined by the inclusion $f_{i}$ of $S^{0+i}=S^{i}$ into the copy of $S^{i} \subset E_{i} \backslash \Sigma E_{i-1}$. There is certainly no $\zeta \in \bar{E}_{0}(X)$ available such that $\varphi \zeta=\left\{\zeta_{i}\right\}:$ Assume to the contrary the existence of such a $\zeta$, then there must necessarily exist a universal $l$ such that $f_{i}$ is stably equivalent to $f_{i}^{\prime}$ : $S^{l} \rightarrow E_{l}$.

However this is just not true by construction.

This assures us that the clusteraxiom does not hold for every spectrum $\underline{E} \in$ Spec.

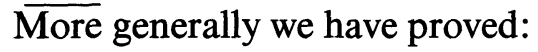

4.5. LEMMA. Let $\underline{E}$ be a $\mathrm{CW}$-spectrum such that the clusteraxiom holds for $\bar{E}_{*}$. Let $X=\mathrm{Cl}_{i=1}^{\infty} X_{i}$ and a family

$$
\tilde{f}_{i}: S^{n+l(i)} \rightarrow E_{l(i)} \bar{\wedge} X_{i}
$$

be given. Then there exists a universal $l$, independent of $i$ such that $\bar{f}_{i}$ is stably homotopic to a

$$
\bar{f}_{i}^{\prime}: S^{n+l} \rightarrow E_{l} \bar{\wedge} X_{i}
$$

Proof. Use the same argument that led to the last conclusion in the preceding third example.

Remark. Proposition 4.4 allows an extension to arbitrary $\Omega$-spectra $\underline{E} \in$ Spec (cf. Theorem 3.1 in [3]). The proof of this assertion follows by Proposition 4.1 and a considerable refinement of the arguments that led to Proposition 4.2. In [3] this is needed as the main tool for comparing $\bar{E}_{*}$ and ${ }^{s} \underline{E}_{*}$ (cf. [8]) on the category $\mathrm{Com}^{f}$. Proposition 4.3 however is, as far as Eilenberg-Mac Lane spectra are concerned, better than Theorem 3.1 in [3] because it works for all (rather, than for finite dimensional) compacta.

5. The Milnor axioms. As we already mentioned J. Milnor characterized an ordinary homology theory on the category $\mathfrak{A}_{\mathrm{CM}}$ of compact pairs (this is J. Milnor's original terminology) by means of the following axioms:

(A1) The clusteraxiom for the subcategory Com $\subset \mathfrak{A}_{\mathrm{CM}}$ (the category of based compact metric spaces).

The preceding section was entirely devoted to this axiom.

(A2) The strong excision axiom which requires that for a homology theory $\left\{H_{n}(), \partial\right\}$ the projection $p:(X, A) \rightarrow(X / A, *)$ induces an isomorphism

$$
H_{*}(X, A) \rightarrow H_{*}(X / A, *)
$$


(A3) The exactness axiom.

(A4) The homotopy axiom.

Contrary to J. Milnor's treatment, we do not have a dimension axiom.

5.1. Definition. A functor $H_{*}: \mathfrak{A}_{\mathrm{CM}} \rightarrow \mathrm{Ab}^{\mathrm{Z}}$ together with the corresponding boundary operators is called a homology theory on $\mathfrak{A}_{\mathrm{CM}} H_{*}=$ $\left\{H_{n}, \partial\right\}$ whenever (A1)-(A4) are satisfied.

It is well-known how to transform such a homology theory into a reduced homology theory on Com and vice-versa. We will freely make use of this correspondence and call a functor $H_{*}$ : Com $\rightarrow \mathrm{Ab}^{\mathrm{Z}}$ (together with natural transformations $\sigma: H_{n} \rightarrow H_{n+1} \Sigma$ ) a homology theory on Com, whenever the related functor on $\mathfrak{A}_{\mathrm{CM}}$ fulfills 5.1.

To this corresponds the concept of a natural transformation $\varphi$ : $H_{*} \rightarrow H^{\prime}{ }_{*}$ between homology theories (cf. Definition 1.3).

This terminology should not be mixed up with that of 1.3 where we dealt with singular homology theories on a shape category.

Let $\underline{E} \in \mathrm{Spec}$ be any spectrum, then the functor $h:$ Com $\rightarrow \overline{\text { Com }}$ gives rise to a homology theory $\underline{E}_{*} h:$ Com $\rightarrow \mathrm{Ab}^{\mathrm{z}}$. According to our custom not to write down the functor $h$ explicitly, we will henceforth write $\bar{E}_{*}$ instead of $\underline{E}_{*} h$.

In [4] $\$ 3$ we have proved for $\underline{E}_{*}, \underline{E} \in$ Spec, all the axioms (A2)(A4). Concerning strong excision, recall that $\bar{E}_{n}(X, A)$ is defined as $\bar{E}_{n}(X \cup C A, *)$, where * denotes the vertex of the cone $C A$. However since every inclusion in Com is a cofibration ([4] Proposition (A9) in $\overline{\mathrm{Com}}$, we have a homotopy equivalence

$$
X \cup C A \simeq X / A \text { in } \overline{\mathrm{Com}} .
$$

This proves (1) for $\underline{E}_{*}$.

The preceding section was devoted to a treatment of the cluster axiom, so that we are able to summarize:

5.2. THEOREM. On the category Com there exists to each $C W$ spectrum $\underline{E}$ a functor $\bar{E}_{*}: \mathrm{Com} \rightarrow \mathrm{Ab}^{\mathrm{z}}$ (together with a natural transformation $\sigma:$ $\underline{\bar{E}}_{n}(X) \rightarrow \bar{E}_{n+1}(\overline{\Sigma X))}$ such that the axioms (A2)-(A4) of a homology theory on Com are satisfied.

The main objective of this section is to establish the following

5.3. ThEOREM. Let $H_{*}, H^{\prime}$ : $\mathrm{Com} \rightarrow \mathrm{Ab}^{\mathrm{Z}}$ be two homology theories on Com and $\alpha: H_{*} \approx H^{\prime}$ be an isomorphism on the subcategory $\underline{P}_{0} \subset$ Com of compact (based) $C W$-spaces. Then there exists a unique extension of $\alpha$ to an isomorphism $\tilde{\alpha}$ : $H_{*} \approx H^{\prime}{ }_{*}$ over Com. 
The proof is preceded by some remarks and two lemmas:

(1) We write $H_{*}$ also for the corresponding homology theory on $\mathfrak{A}_{\mathrm{CM}}$. The isomorphism $\alpha$ can be extended to an isomorphism (also denoted by the same letter) between $H_{*}$ and $H^{\prime}{ }_{*}$ defined on the category of polyhedral pairs.

(2) Instead of working with actual CW-spaces (resp. CW-pairs or polyhedral pairs), we will use ANR subspaces of a Hilbert cube $Q$ in which any space $X \in$ Com is supposed to be embedded. This does clearly not cause any difficulties.

(3) Let $\bar{\zeta} \in H_{n}(X)$ be any element. A simple exactness argument guarantees that $H_{n+1}(Q, X) \stackrel{\partial}{\approx} H_{n}\left(X, x_{0}\right)$, hence there exists a uniquely determined $\zeta \in H_{n+1}(Q, X)$ such that $\partial \zeta=\bar{\zeta}$. It is actually this $\zeta$ with which we are going to operate.

(4) Let $(X, A),\left(X_{1}, A_{1}\right),\left(X_{2}, A_{2}\right) \in \mathfrak{A}_{\mathrm{CM}}$ be three pairs such that $X=X_{1} \cup X_{2}, X_{1} \cap X_{2}=A_{1} \cap A_{2}$.

Denote by

$$
i:(X, A) \subset\left(X, A_{1} \cup A_{2} \cup A\right), \quad i_{j}:\left(X_{j}, A_{J}\right) \subset\left(X, A_{1} \cup A_{2} \cup A\right)
$$

the inclusions. Let $\zeta_{i} \in H_{m}\left(X_{i}, A_{i}\right)$ be two fixed elements, then we denote by $\zeta=\zeta_{1} \oplus \zeta_{2} \in H_{m}(X, A)$ any element, having the property

$$
i_{*} \zeta=i_{1 *} \zeta_{1}+i_{2 *} \zeta_{2} \text {. }
$$

This $\zeta$ may not exist nor is it necessarily uniquely determined. We can of course extend this terminology to finitely many summands.

(5) The cluster axiom enables us to extend this construction to countably many summands:

Let to this end $Q$ be the Hilbert cube, $X \subset Q$ a given compact space and let $\bar{\zeta} \in H_{n}(X), \zeta \in H_{n+1}(Q, X)$ with $\partial \zeta=\bar{\zeta}$ be fixed elements. We take a decreasing sequence $P_{1}=Q, P_{2} \supset \cdots P_{k} \supset \cdots$ of compact ANRs with $\cap P_{i}=X$. Moreover we establish an increasing sequence of compact ANRs $\varnothing=U_{1} \subset U_{2} \subset \cdots$ in $Q$ having the following properties:

(1) $U_{i} \cap P_{i+k}=\varnothing$ for $k \geq 1$, (2) $\overline{P_{j} \backslash P_{i}} \subset$ Int $U_{i}, i>j$. All this can be obviously achieved.

We set:

$$
\begin{aligned}
& R_{i}=P_{i} \cap U_{i}, \quad X_{i}=P_{i} \cap U_{i+1}, \quad A_{i}=R_{i} \cup R_{i+1} \quad \text { (Fig. 1). } \\
& \bar{R}_{\imath}=R_{i} \cup X, \quad \bar{X}_{i}=X_{i} \cup X, \quad \overline{A_{i}}=A_{i} \cup X .
\end{aligned}
$$




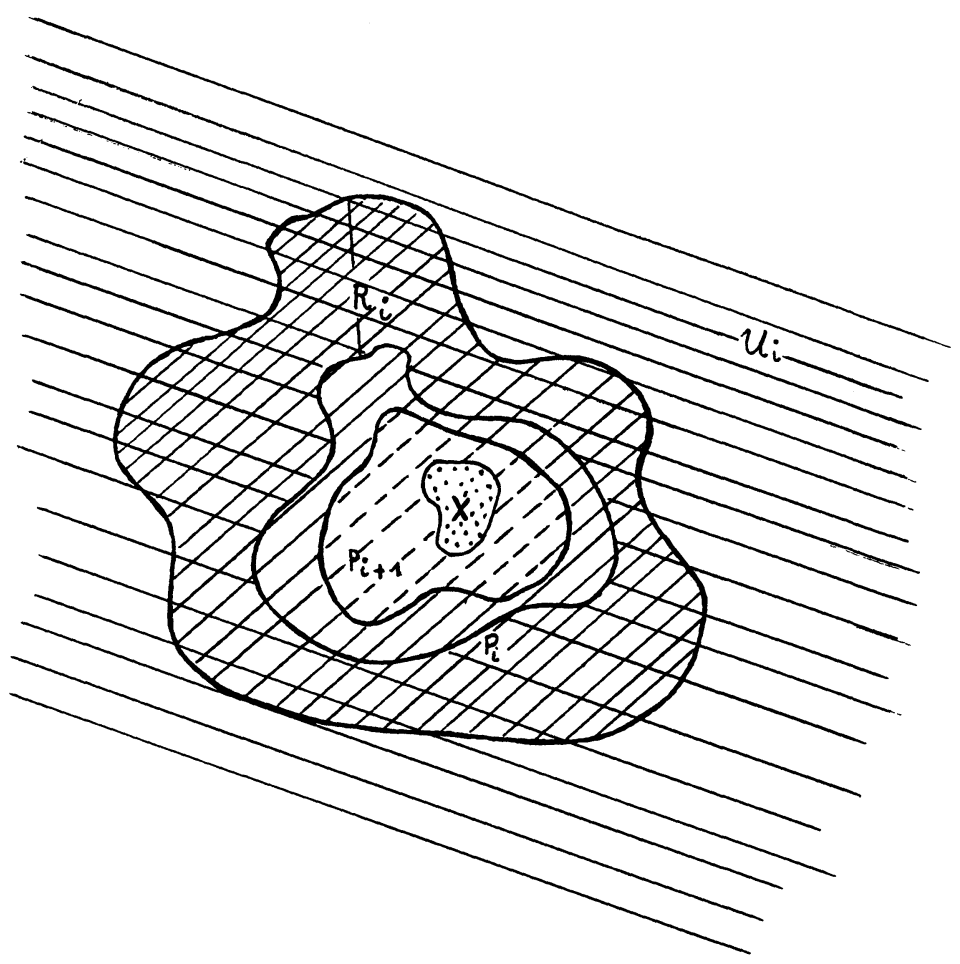

Figure 1

Let $\zeta_{i} \in H_{m}\left(X_{i}, A_{i}\right)$ for all $i$ be given elements and $\bar{\zeta}_{i}$ the images of $\zeta_{i}$ under the inclusion $\left(X_{i}, A_{i}\right) \subset\left(\bar{X}_{i}, \bar{A}_{i}\right)$. We assume:

(*) The existence of elements $\eta_{r} \in H_{m}\left(X_{1} \cup \cdots \cup X_{r}, R_{r+1}\right)$ which are inductively determined

$$
\zeta_{1}=\eta_{1}, \eta_{2}=\eta_{1} \oplus \zeta_{2}, \ldots, \eta_{r}=\eta_{r-1} \oplus \zeta_{r},
$$

and denote by $\bar{\eta}_{r} \in H_{m}\left(\bar{X}_{1} \cup \cdots \cup \bar{X}_{r}, \bar{R}_{r+1}\right)$ the image of $\eta_{r}$ under the corresponding inclusion.

Since we have

$$
R_{i} \cap R_{i+1}=\varnothing,
$$

the definition of $\left(X_{i}, A_{i}\right)$ implies that there exists $\rho_{i}^{(1)}, \rho_{i}^{(2)} \in H_{m-1}\left(R_{i}\right)$ such that

$$
\rho_{i}^{(1)}-\rho_{i+1}^{(2)}=\partial \zeta_{i},
$$

where we omitted the inclusions $R_{i} \subset A_{i}$ from our notation. Because $R_{1}=Q \cap U_{1}=\varnothing$, we have $\rho_{1}^{(1)}=\rho_{1}^{(2)}=0$.

The existence of $\eta_{r}$ implies that we have

$$
\rho_{i}^{(1)}=\rho_{i}^{(2)} .
$$


The following lemma asserts that we are able to find a $\zeta \in H_{m}(Q, X)$ $=H_{m}\left(\bigcup_{i=1}^{\infty} \bar{X}_{t}, X\right)$ which can be considered as some kind of $\eta_{\infty}$ :

5.4. Lemma. Assume that (*) is satisfied, then (1) there exists a $\zeta \in H_{m}(Q, X)$ such that

$$
k_{r *} \zeta=l_{r *} \bar{\eta}_{r}
$$

with inclusions

$$
\begin{aligned}
& k_{r}:(Q, X) \subset\left(Q, \bigcup_{i=r+1}^{\infty} \bar{X}_{i}\right) \\
& l_{r}:\left(\bigcup_{i=1}^{r} \bar{X}_{i}, \bar{R}_{r+1}\right) \subset\left(Q, \bigcup_{i=r+1}^{\infty} \bar{X}_{i}\right) ;
\end{aligned}
$$

(2) any other elements $\zeta^{\prime}$ satisfying (2) (with possibly different family $\left\{\bar{\zeta}_{r}\right\}$ and same $\left.\left\{\zeta_{l}\right\}\right)$ is equal to $\zeta$.

Proof. Let $Z_{t}=\bar{X}_{i} / \bar{A}_{i}$; form the cluster of different copies of the spaces $\bar{X}_{i}$ and define

$$
Z=\mathrm{Cl}\left(\bar{X}_{i} / X\right)
$$

Observe that $\bar{X}_{i} / X=X_{i} \cup X / X=X_{t}^{+}$and $\bar{A}_{i} / X=A_{\imath} \cup X / X=$ $A_{i}^{+}$.

Due to (A2) we have an isomorphism

$$
H_{m}\left(\mathrm{Cl} Z_{l}, *\right) \approx H_{m}\left(Z, \mathrm{Cl} \overline{A_{i}} / X\right),
$$

while (A1) provides us with a $\eta^{\prime} \in H_{m}\left(\mathrm{Cl} Z_{i}, *\right)$, satisfying $\eta^{\prime} \mid \bar{X}_{i} / \overline{A_{i}}=$ $\zeta_{i}^{\prime} \in H_{m}\left(\bar{X}_{l} / \overline{A_{l}}\right) \approx H_{m}\left(\bar{X}_{i}, \overline{A_{i}}\right)$ where $\zeta_{l}^{\prime}$ corresponds to $\bar{\zeta}_{l}$ under this isomorphism.

Here we denote by $\eta^{\prime} \mid \bar{X}_{i} / \bar{A}_{i}$ the $i$ th factor in the element corresponding to $\eta^{\prime}$ by the isomorphism (A1):

$$
H_{m}\left(\underset{1}{\mathrm{C}} \mathrm{l} Z_{l}, *\right) \approx \prod_{i=1}^{\infty} H_{m}\left(Z_{i}, *\right) .
$$

To $\eta^{\prime}$ there exists under the isomorphism (4) a $\eta \in H_{m}\left(Z, \mathrm{Cl}_{1}^{\infty} A_{i}^{+}\right)$. We have an infinite folding map:

$$
f:\left(Z, \stackrel{\infty}{\mathrm{C}} A_{i}^{+}\right) \rightarrow\left(Q / X, \cup A_{i}^{+}\right)
$$

(identifying back the different copies of the $X_{i}$ as subsets of $Q$ resp. for the quotient spaces $Q / X)$ and obtain $\eta^{\prime \prime}=f_{*} \eta \in H_{m}\left(Q / X, \cup_{l=1}^{\infty} A_{l}^{+}\right)$.

$(* *)$ There exists an element $\zeta \in H_{m}(Q, X) \approx H_{m}(Q / X, *)$ such that $j_{*} \zeta=\eta^{\prime \prime}, j:(Q / X, *) \stackrel{\subset}{\rightarrow}\left(Q / X, \cup A_{i}^{+}\right)$. 
Proof. Because of the exact (reduced) homology sequence of the pair $\left(Q / X, \cup_{i=1}^{\infty} A_{i}^{+}\right)$it suffices to prove that $\partial \eta^{\prime \prime}=0$. To this end we have the commutative diagram

$$
\begin{aligned}
& \prod_{i=1}^{\infty} H_{m}\left(\bar{X}_{i} / \overline{A_{i}}\right) \quad \stackrel{\Delta}{\rightarrow} \quad \prod_{i=1}^{\infty} H_{m-1}\left(A_{i}^{+}\right) \\
& \| \downarrow \varphi
\end{aligned}
$$

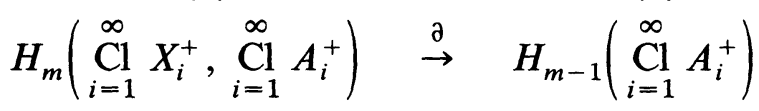

$$
\begin{aligned}
& \downarrow f_{*} \\
& H_{m}\left(Q / X, \bigcup_{i=1}^{\infty} A_{i}^{+}\right) \quad \stackrel{\partial}{\rightarrow} H_{m-1}\left(\bigcup_{i=1}^{\infty} A_{i}^{+}\right)
\end{aligned}
$$

where the vertical isomorphisms stem from (A1) (or from (A1) in combination with (A2)) and the vertical arrows $f_{*}$ are induced by the folding $\operatorname{map} f$.

According to (5) we have proved $\partial \eta^{\prime \prime}=0$ whenever we are able to confirm that $f_{*} \varphi\left\{\Delta \zeta_{i}^{\prime}\right\}=0 \in H_{m-1}\left(\cup_{i=1}^{\infty} A_{i}^{+}\right)$, where $\Delta: H_{m}\left(\bar{X}_{i} / \bar{A}_{i}\right) \rightarrow$ $H_{m-1}\left(A_{i}^{+}\right)$is the composite of $\partial: H_{m}\left(X_{i}^{+}, A_{i}^{+}\right) \rightarrow H_{m-1}\left(A_{i}^{+}\right)$with the natural isomorphism

$$
H_{m}\left(\bar{X}_{i} / \bar{A}_{i}\right) \approx H_{m}\left(X_{i}^{+}, A_{i}^{+}\right) .
$$

On the other hand we have $A_{i}=R_{i} \cup R_{i+1}$. Hence the space $R_{i}$ appears in the cluster $\mathrm{Cl}_{i=1}^{\infty} A_{i}^{+}$twice, namely as a subset $R_{i}^{(1)} \subset A_{i}$ and secondly as a subset $R_{i}^{(2)}$ of $A_{i-1}$. The folding map $f$ identifies both copies. We have already detected the elements $\rho_{i}^{(k)} \in H_{m-1}\left(R_{i}^{(k)}\right), k=1,2$ such that in $Q$ (2) (now for $A_{i}^{+}$instead of $A_{i}$ ) is satisfied.

We can rearrange the cluster

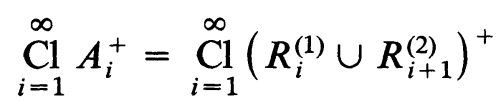

getting a new cluster $C=\mathrm{Cl}_{i=1}^{\infty}\left(R_{i}^{(1)} \cup R_{i}^{(1)}\right)^{+}$with folding map $g$ : $C \rightarrow$ $\bigcup_{i=1}^{\infty} A_{i}^{+}$.

Moreover there exist isomorphisms in a commutative diagram

$$
\begin{aligned}
& \prod_{i=1}^{\infty} H_{m-1}\left(R_{i}^{(1)} \cup R_{i}^{(2)}\right) \quad \approx \quad \prod_{i=1}^{\infty} H_{m-1}\left(A_{i}^{+}\right) \\
& \text {\& } 1 \\
& H_{m-1}(C) \\
& \approx \quad H_{m-1}\left(\stackrel{\infty}{\mathrm{C}}_{i=1}^{\varphi} A_{i}^{+}\right) \\
& g_{*} \searrow \\
& \swarrow f_{*} \\
& H_{m-1}\left(\bigcup_{i=1}^{\infty} A_{i}^{+}\right)
\end{aligned}
$$


As a result we have

$$
0=g_{*} \psi\left\{\rho_{i}^{(1)}-\rho_{i}^{(2)}\right\}=f_{*} \varphi\left\{\rho_{i}^{(1)}-\rho_{i+1}^{(2)}\right\}=\partial \eta^{\prime \prime} .
$$

This completes the construction of $\zeta \in H_{m}(Q, X)$.

Condition (3) asserts that $\bar{\eta}_{r}=l_{r}^{-1}{ }_{*} k_{r *} \zeta$ (since $l_{r}$ is easily recognized to be an excision) and therefore simply states that by replacing infinite clusters by finite ( $r$-fold) wedges we get back $\eta_{r}$. The verification of this fact is straightforward.

This completes the proof of the assertion (1) in 5.4.

We accomplish a proof of 5.4 (2) by collecting the following remarks:

(1) the inclusion

$$
\left.j:\left(\bigcup_{i=1}^{\infty} \overline{A_{i}}, x_{0}\right) \subset\left(\bigcup_{i=1}^{\infty} \overline{A_{i}}, X\right)\right) \quad\left(\text { resp. } j_{k}:\left(\bigcup_{1}^{k} \overline{A_{i}}, x_{0}\right) \subset\left(\bigcup_{1}^{k} \overline{A_{i}}, X\right)\right)
$$

induce an epimorphism in homology.

Proof. We have $X \cap A_{i}=\varnothing$ and therefore a commutative diagram

$$
\begin{array}{rc}
H_{m}\left(\bigcup_{1}^{\infty} \overline{A_{i}}, x_{0}\right) \stackrel{j_{*}}{\rightarrow} & H_{m}\left(\bigcup_{1}^{\infty} \overline{A_{i}}, X\right) \\
& { }_{\rho_{*} \nwarrow} \\
& H_{m}\left(\bigcup_{1}^{\infty} A_{i}^{+}, *\right)
\end{array}
$$

with inclusion $\rho$ where the vertical arrow is an excision.

(2) The sequence $\left\{\zeta_{i}\right\}$ determines $\eta^{\prime \prime}$ uniquely. Let $\zeta, \zeta^{\prime} \in H_{m}(Q / X, *)$ $=H_{m}(Q, X)$ be two elements which map into $\eta^{\prime \prime}$ in

$$
H_{m}(Q / X, *) \rightarrow H_{m}\left(Q / X, \bigcup_{1}^{\infty} A_{i}^{+}\right)
$$

then by exactness, $\zeta-\zeta^{\prime}=q_{*} \alpha$, with $\alpha \in H_{m}\left(\cup_{1}^{\infty} A_{i}^{+}\right), q$ : $\left(\cup_{1}^{\infty} A_{i}^{+}, x_{0}\right) \subset$ $(Q / X, *)$.

Consider the diagram

$$
\begin{array}{ccccc} 
& H_{m}\left(\bigcup_{1}^{\infty} A_{i}^{+}, *\right) \\
H_{m}\left(\bigcup \overline{A_{i}}, x_{0}\right) & \stackrel{j_{*}}{\leftarrow} & H_{m}\left(\bigcup_{1}^{\infty} \overline{A_{i}}, X\right) & \rightarrow & H_{m}(Q, X) \\
\partial^{\prime \prime} \downarrow & & \partial^{\prime} \downarrow & & \partial \downarrow \\
H_{m-1}\left(x_{0}\right) & \leftarrow & H_{m-1}\left(X, x_{0}\right) & \approx & H_{m-1}\left(X, x_{0}\right)
\end{array}
$$


whose commutativity ensures that $\partial \alpha=\partial \zeta-\partial \zeta^{\prime}=0$. This proves that two $\zeta, \zeta^{\prime}$ which are achieved by our construction satisfy $\partial \zeta=\partial \zeta^{\prime}$.

(3) Let $\zeta \in H_{m}(Q, X)$ be given such that for fixed $\left\{\zeta_{i}\right\}$ and some selection of $\left\{\bar{\eta}_{r}\right\}(3)$ holds. Observe that $l_{r}$ is an excision, hence $l_{r *}$ an isomorphism. We have a commutative diagram for a $\zeta \in H_{m}(X, Q)$ such that (3) (for all $r$ ) holds:

$$
\begin{aligned}
& \zeta \in H_{m}(Q, X) \\
& \text { I " } \\
& \bar{\zeta} \in H_{m}(Q / X) \quad \rightarrow \quad H_{m}\left(Q / X, \bigcup_{i=1}^{\infty} A_{i}^{+}\right) \ni \eta^{\prime \prime} \\
& \prod_{i=1}^{\infty} H_{m}\left(\bar{X}_{i}, \bar{A}_{i}\right) \ni\left\{\bar{\zeta}_{i}\right\}
\end{aligned}
$$

which guarantees that $\zeta$ is in fact obtained by the construction process which we established during the proof of (1). These remarks ensure that we have $\partial \zeta=\partial \zeta^{\prime}$ for any other $\zeta^{\prime}$ which satisfies (3) (using an eventually different $\left\{\bar{\eta}_{r}\right\}$ related to the same family $\left.\left\{\zeta_{i}\right\}\right)$. Now $\zeta=\zeta^{\prime}$ follows by the fact that $\partial: H_{n+1}(Q, X) \approx H_{n}\left(X, x_{0}\right)$ is an isomorphism (cf. Remark (3) at the beginning of this proof).

We can of course get back the $\eta_{r}$ and even the $\zeta_{i}$ from $\zeta$ (which in analogy to the finite case will be denoted by $\zeta=\oplus_{i=1}^{\infty} \zeta_{i}$ ).

5.5. Lemma. Let $(Q, X),\left(X_{i}, A_{i}\right)$ be as before and fix a $\zeta \in H_{m}(Q, X)$. Then there exists $a \zeta_{i} \in H_{m}\left(X_{i}, A_{i}\right)$ for all $i$, such that

$$
\zeta=\bigoplus_{i=1}^{\infty} \zeta_{i} \text {. }
$$

Proof. We have $\zeta_{j} \in H_{m}\left(Q, \cup_{i \neq j} X_{i}\right)$ for any $j$ and obtain a $\zeta_{j} \in$ $H_{m}\left(X_{j}, A_{j}\right)$ be excision. Also the elements $\eta_{r}$ are obtained by excision from $\eta_{r}^{\prime} \in H_{m}\left(Q, \cup_{i=r}^{\infty} X_{i} \cup X\right)$. The verification of (2) is now immediate.

Proof of Theorem 5.3. The basic idea of the proof of Theorem 5.3 is this: Starting with a $\bar{\zeta} \in H_{m-1}\left(X, x_{0}\right)$ we find a $\zeta \in H_{m}(Q, X)$ which can be cut into pieces $\left\{\zeta_{i}\right\}$ by means of Lemma 5.5. On the other hand Lemma 5.4 allows us to paste together these pieces $\left\{\zeta_{i}\right\}$ (which are required to satisfy certain compatibility conditions) to the effect that we get a $\zeta \in H_{m}(Q, x)$ and finally a $\partial \zeta=\bar{\zeta} \in H_{m-1}\left(X, x_{0}\right)$. While $\bar{\zeta} \in H_{m}\left(X, x_{0}\right)$ is eventually defined outside $\underline{P}_{0}$, the pieces $\zeta_{i}$ are all defined for pairs in $\underline{P}_{0}^{2}$ and therefore accessible to the transformation $\alpha$. 
Let $\bar{\zeta} \in H_{n}(X), \zeta \in H_{n+1}(Q, X)$ be given, such that $\partial \zeta=\bar{\zeta}$. We write for simplicity $H_{*}(X)\left(H^{\prime}{ }_{*}(X)\right)$ instead of $H_{*}\left(X, x_{0}\right)\left(H_{*}^{\prime}\left(X, x_{0}\right)\right)$; all homology groups in this proof are understood to be reduced.

Lemma 5.5 yields a sequence $\left\{\zeta_{i} \in H_{n+1}\left(X_{i}, A_{i}\right)\right\}$ such that $\zeta=$ $\oplus_{i=1}^{\infty} \zeta_{i}$. This enables us to extend $\alpha: H_{*} \approx H^{\prime}$ in the following way: Let $\bar{\zeta} \stackrel{i=H_{n}}{\epsilon}(X)$ be given, then we go over to $H_{n+1}(Q, X)$ and the family $\left\{\zeta_{i}\right\}$. Because all $\zeta_{i}$ are defined for spaces in a subcategory where $\alpha$ has been already established, we have the family $\left\{\zeta_{i}\right\}$ (see Remarks (1), (2) at the beginning of this proof) which in turn establishes (due to Lemma 5.4) again an element $\tilde{\alpha} \bar{\zeta} \in H_{n}^{\prime}(X)$. By Remark (3) and the last conclusion of Lemma 5.4 , this element $\tilde{\alpha} \bar{\zeta}$ is uniquely determined by $\bar{\zeta}$ (because every intermediate step has this property, with fixed $P_{i}, U_{i}$ ).

Observe that we can instead of a family $\left\{\zeta_{i}\right\}$ equally well deal with a related family $\left\{\tilde{\eta}_{r} \in H_{n+1}\left(Q, P_{r}\right)\right\}$ which determines (1) the family $\left\{\eta_{r} \in\right.$ $\left.H_{n+1}\left(\cup_{i=1}^{r} \bar{X}_{i}, \bar{R}_{r+1}\right)\right\}$ by excision and finally (2) a family $\left\{\zeta_{i} \in\right.$ $\left.H_{n+1}\left(\bar{X}_{i}, \bar{A}_{i}\right)\right\}$. Since $\left\{\zeta_{i}\right\}$ determines $\bar{\zeta}=\partial \zeta$ uniquely, we are naturally led to the question under what conditions two different families $\left\{\tilde{\eta}_{r} \in\right.$ $\left.H_{n+1}\left(Q, P_{r}\right)\right\}, \quad\left\{\tilde{\eta}_{r}^{\prime} \in H_{n+1}\left(Q, P_{r}^{\prime}\right)\right\} \quad$ (with a different approximating $\left\{P_{i}^{\prime}, U_{i}^{\prime}\right\}$ ) determine the same $\zeta$ : This problem is settled by means of the following observation:

(F1) Let $\{l(r)\}$ be an infinite subsequence of the integers, then $\left\{\tilde{\eta}_{r}\right\}$ and $\left\{\tilde{\eta}_{l(r)}\right\}$ determine obviously the same $\zeta$.

(F2) Assume that $\left\{\tilde{\eta}_{r}\right\},\left\{\tilde{\eta}_{r}^{\prime}\right\}$ have the property that to each $r$ there exists an index $l(r)$ such that $P_{l(r)}^{\prime} \subset P_{r}$ and $j_{*} \tilde{\eta}_{l(r)}^{\prime}=\tilde{\eta}_{r}\left(j:\left(Q, P_{l(r)}^{\prime}\right) \subset\right.$ $\left.\left(Q, P_{r}\right)\right)$, then $\left\{\tilde{\eta}_{r}\right\}$ and $\left\{\tilde{\eta}_{r}^{\prime}\right\}$ determine the same $\zeta$.

(F3) Let on the other hand $\zeta$ be determined by $\left\{\tilde{\eta}_{r}\right\},\left(\tilde{\zeta}^{\prime}\right.$ by $\left.\left\{\tilde{\eta}_{r}^{\prime}\right\}\right)$ and $\zeta=\zeta^{\prime}$, then we can find to each $P_{r}$ an index $l(r)$ as in (F2) such that $k_{*} \zeta=\tilde{\eta}_{r}=j_{*} k_{*}^{\prime} \zeta=j_{*} \tilde{\eta}_{l(r)}^{\prime}$, with inclusions $k:(Q, X) \subset\left(Q, P_{r}\right), \quad k^{\prime}$ : $(Q, X) \subset\left(Q, P_{l(r)}^{\prime}\right)$.

As a result we have that $\zeta=\zeta^{\prime}$ if and only if (F2) holds.

The assignment $\tilde{\alpha}$ can be defined by using the families $\left\{\tilde{\eta}_{r}\right\}$ instead of $\left\{\zeta_{i}\right\}$ : We have the family $\left\{\alpha \tilde{\eta}_{r}\right\}$ which in turn determines a family $\left\{\zeta_{i}^{\prime} \in H_{n+1}^{\prime}\left(\bar{X}_{i}, \bar{A}_{i}\right)\right\}$ and finally the element $\tilde{\alpha} \bar{\zeta}$.

These remarks enable us to prove

(a) $\tilde{\alpha}$ is natural and compatible with suspensions.

Proof. Let $f:\left(X, x_{0}\right) \rightarrow\left(Y, y_{0}\right)$ be continuous. We can extend $f$ to a $F:(Q, X) \rightarrow(Q, Y)$. Denoting the corresponding sequence of approximating ANRs by $P_{1}^{\prime}=Q \supset P_{2} \supset \cdots, \cap_{i=1}^{\infty} P_{i}^{\prime}=Y$, there corresponds to 
each index $i$ a minimal $l(i)$ such that $F\left(P_{i}\right) \subset P_{l(i)}^{\prime}$. We have $F^{\prime}$ : $\left(Q, P_{i}\right) \rightarrow\left(Q, P_{l(i)}^{\prime}\right)$. So we can assign to each $\tilde{\eta}_{r}$ a $\tilde{\eta}_{l(r)}^{\prime}=F_{*}^{\prime} \tilde{\eta}_{r} \in$ $H_{n+1}\left(Q, P_{l(i)}^{\prime}\right)$, in such a way that $\left\{\tilde{\eta}_{l(r)}^{\prime}\right\}$ is a family which determines $f_{*} \zeta$. The naturality of $\tilde{\alpha}$ requires the commutativity of the diagram

$$
\begin{array}{ccc}
H_{n}(X) & \stackrel{\tilde{\alpha}}{\rightarrow} & H_{n}^{\prime}(X) \\
f_{*} \downarrow & & \cdot \\
H_{n}(Y) & \stackrel{\tilde{\alpha}}{\rightarrow} & H_{n}^{\prime}(Y) .
\end{array}
$$

Since $\alpha$ itself is supposed to be natural, this can be deduced by looking upon the two chains of correspondences for a fixed $\zeta \in H_{n}(X)$ :

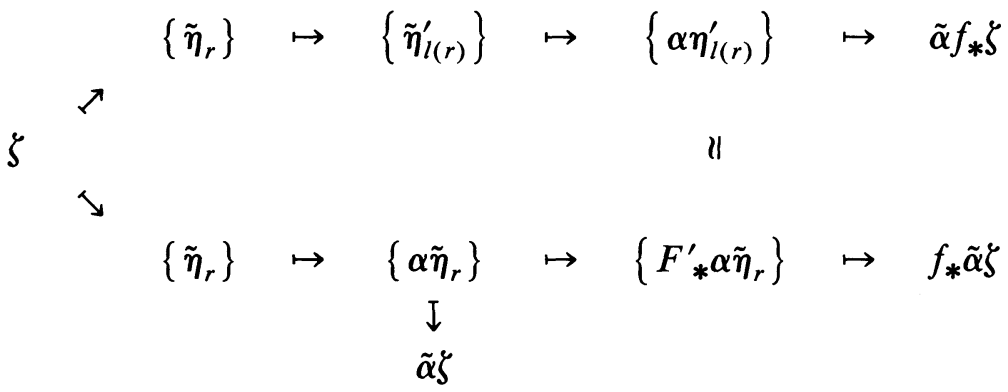

where the vertical " $\simeq$ " indicates a relation of type (F1).

The second assertion in (a) follows immediately by construction.

(b) $\tilde{\alpha}$ is a homomorphism.

Proof. This follows because the whole construction process of $\tilde{\alpha}$ is compatible with the group structure:

Let $\bar{\zeta}_{1}, \bar{\zeta}_{2} \in H_{n}(X)$ be given, then we have $\partial\left(\zeta_{1}+\zeta_{2}\right)=\bar{\zeta}_{1}+\bar{\zeta}_{2}$, moreover $\left\{\eta_{r}^{(1)}+\eta_{r}^{(2)}\right\}$ is a family for $\zeta_{1}+\zeta_{2}$ (in the sense of the preceding construction) etc.

(c) $\tilde{\alpha}$ is by $\alpha$ uniquely determined.

Proof. Let $\beta: H_{n}(X) \rightarrow H_{n}^{\prime}(X)$ be another extension of $\alpha$, then for given $\bar{\zeta} \in H_{n}(X)$, the family $\left\{\beta \tilde{\eta}_{r}\right\}$ is easily seen to determine $\beta \bar{\zeta} \in$ $H_{n}^{\prime}(X)$. Since $\alpha=\beta$ for all $\tilde{\eta}_{r}$, we have $\left\{\alpha \tilde{\eta}_{r}\right\}=\left\{\beta \tilde{\eta}_{r}\right\}$, where, by definition, the first family determines $\tilde{\alpha} \bar{\zeta}$ and the second one $\beta \bar{\zeta}$. Hence (c) follows.

(d) $\tilde{\alpha}$ is an isomorphism. 
Proof. Follows directly from (c) or can be seen by putting $\alpha^{-1}$ instead of $\alpha$ into the construction process of $\tilde{\alpha}$.

This completes the proof of 5.3.

We could of course easily adjust the preceding proof to establish the following slightly more general result:

5.6. Corollary. Let $H_{*}, H^{\prime}{ }_{*}$ be homology theories on Com (in the sense of Definition 5.1) $\alpha: H_{*} \rightarrow H^{\prime}{ }_{*}$ a natural transformation on $\underline{P}_{0}$, then there exists a unique extension $\tilde{\alpha}: H_{*} \rightarrow H_{0}^{\prime}$ of $\alpha$ over Com.

Concerning the relations to $\overline{\mathrm{Com}}$ we have:

5.7. Corollary. Let (1) $H_{*}: \mathrm{Com} \rightarrow \mathrm{Ab}^{\mathrm{z}}$ be any homology theory on

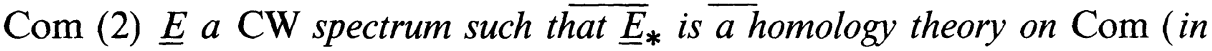
the sense of Definition 5.1, which implies in particular that the cluster axiom holds) (3) $\alpha: H_{*} \approx \underline{E}_{*}$ a natural isomorphism on the category $\underline{P}_{0}$. Then there exists an extension $\bar{H}_{*}: \overline{\mathrm{Com}} \rightarrow \mathrm{Ab}^{\mathrm{z}}$ of $H_{*}$ over the shape category $\overline{\mathrm{Com}}$ and an isomorphism $\tilde{\alpha}: H_{*} \approx \overline{\bar{E}_{*}}$.

This is an immediate consequence of 5.2 and 5.3.

In the next section we need the category $\mathrm{Com}^{f}$ of finite dimensional compact metric spaces. The proof of 5.3 immediately carries over to this case, so that we can restate $5.3,5.6,5.7$ for $\mathrm{Com}^{f}$.

5.8. THEOREM. The conclusions of 5.3, 5.6, 5.7 are still valid after replacing Com by $\mathrm{Com}^{f}$.

REMARK. As pointed out in [3] p. 209 (proof of Theorem 3.2), the arguments which lead to the construction of $\tilde{\alpha}$ (in the course of the proof of Theorem 5.3) are still valid whenever only the homology theory $H^{\prime}{ }_{*}$ (but not necessarily $H_{*}$ ) satisfies a clusteraxiom: We need the clusteraxiom solely to paste together a sequence $\left\{\alpha \zeta_{i}\right\}$ (or alternatively $\left\{\alpha \tilde{\eta}_{r}\right\}$ ) in order to obtain a $\tilde{\alpha} \bar{\zeta}$. The clusteraxiom is not needed to break a given element $\bar{\zeta}$ into appropriate pieces $\left\{\zeta_{i}\right\}$.

6. Applications. We collect together some consequences of the preceding existence and uniqueness theorems. Some of these results are already known, but with different proofs. 
We summarize some of the results of $\S \S 1-3$ in:

6.1. THEOREM. Let $E$ be an $C W$ spectrum, then the homology theory $\underline{E}_{*}: \underline{P}_{1 h} \rightarrow \mathrm{Ab}^{\mathrm{Z}}$ allows, up to an isomorphism, a unique extension $\underline{E}_{*}$ : $\underline{\bar{K}}_{h} \rightarrow \mathrm{Ab}^{\mathrm{Z}}, \overline{\tilde{E}}_{*}()=\underline{E}_{*}(|\bar{S}()|)$, such that the Whitehead axiom $\left.\underline{\mathrm{W}}\right)$ (Definition 1.2) holds. Moreover $\tilde{E}_{*}$ is of compact support (Definition 1.7). For $\underline{E} \in \mathrm{CSpec}$ and $X$ being an s-continuum, one has a natural isomorphism $\underline{E}_{*}(X) \approx \overline{\tilde{E}_{*}(X)}$.

Proof. This follows immediately from 1.8, 2.2, 3.3.

6.2. TheOREM. For any abelian group $G$, Steenrod-Sitnikov homology theory $H_{*}^{S}(; G)$ turns out to be naturally isomorphic to $\overline{K(G)}_{*}($ ) on the category $\mathrm{Com}$. Hence $H_{*}^{S}(;, G)$ can be uniquely extended over $\overline{\mathrm{Com}}$.

Proof. As well $H_{*}^{S}(; G)$ as $\overline{K(G)}_{*}()$ are fulfilling the Milnor axioms $\S 5$ (A1)-(A4): As far as $H_{*}^{S}(; \bar{G})$ is concerned this can be found in [10], while 4.3 and 5.2 together confirm that for $\overline{K(G)}_{*}\left(\right.$ ). Since $H_{*}^{S}(; G)=$ $H_{*}(; G)$ and $\overline{K(G)_{*}}(\mathrm{l})$ are isomorphic on $\underline{P}_{0}$, Theorem 6.2 can be immediately deduced from 5.3 and 5.7.

6.3. Corollary. Let $G$ be a finitely generated abelian group, $X$ an $s$-continuum, then there exists a natural isomorphism between $H_{*}(|\bar{S}(X)| ; G)$ and $H_{*}^{S}(X ; G)$.

Proof. This is an immediate consequence of 6.1 and 6.2.

REMARK. The last corollary is a generalization of Theorem 7.7 in [2] (where this was proved by different methods for $G=\mathbf{Z}$ ). Observe that the $s$-connectedness of $X$ is not necessary for 6.2 but enters into our considerations through 6.1 .

6.4. THEOREM. Let $\hat{H}_{*}$ be an ordinary homology theory on Com (i.e. one for which $\$ 5$ (A1)-(A4) and in addition a dimension axiom is valid) with coefficient group $\hat{H}_{0}($ point $) \approx G$. Then $\hat{H}_{*}()$ is naturally isomorphic to Steenrod-Sitnikov homology $H_{*}^{S}(;, G)$.

Proof. By the Eilenberg-Steenrod uniqueness theorem, we have $\hat{H}_{*}() \approx H_{*}(; G)$ on $\underline{P}_{0}$ (resp. on polyhedral pairs). Now the assertion follows by application of 6.2 . 
REMARK. This is nothing else than J. Milnor's uniqueness theorem in [12].

For the sake of completeness we restate Corollary 4.2 in [4] which follows now from 6.1, the fact that $K(G) \in$ CSpec for finitely generated $G$ and homological algebra:

6.5. Corollary. Let $G$ be finitely generated, abelian; $X \in$ Com s-connected (i.e. a s-continuum), then we have a natural universal coefficient sequence:

$$
\left.0 \rightarrow \overline{K(Z)}_{n}(X) \otimes G \rightarrow \overline{\overline{K(G)}}_{n}(X) \rightarrow \operatorname{Tor} \overline{\overline{K(Z)}}_{n-1}(X), G\right) \rightarrow 0 .
$$

REMARK. This result cannot be extended to an arbitrary coefficient groups $G$ (which is not finitely generated) because of a result in [10] asserting that a non-trivial homology theory satisfying a clusteraxiom does not admit a universal coefficient sequence for all abelian $G$. As a consequence we are able to provide for any non-finitely generated $G$ an $s$-continuum $X$ such that

$$
\overline{\bar{K}(G)_{*}}(X) \approx \overline{\overline{K(G)_{*}}}(X) .
$$

As can be easily realized, the $s$-continuum $X=\mathrm{Cl}_{i=1}^{\infty} S_{i}^{2}\left(S_{i}^{2}=S^{2}\right.$ for any $i=1,2, \ldots$ ) has the desired property.

In [6], [8], [9] the authors deal with a functor

$$
\text { Ext: } \underline{\operatorname{Com}}_{h} \rightarrow \underline{\mathrm{Ab}}
$$

which is defined by functional analytic methods. This functor gives rise to a homology theory

$$
\varepsilon_{n}(X)= \begin{cases}\operatorname{Ex}(X) & \text { if } n \equiv 1(2) \\ \operatorname{Ext}(\Sigma X) & \text { if } n \equiv 0(2)\end{cases}
$$

on the category Com which has very far reaching applications in functional analysis.

Concerning the definition of Ext and the verification of its basic properties the reader is referred to [6]. In [5] as well as in [7] the problem of the uniqueness of Steenrod extensions has been raised for the first time.

In [8] Remark 7.7 the authors deduce a non-canonical version of Theorem 5.7 for homology theories with coefficient groups of finite type.

Furthermore it is proved in [6] (Theorem 7.3) that for Ext and consequently for $\varepsilon_{*}$ the clusteraxiom holds.

This makes sense either for the based version of $\varepsilon_{*}$ or by talking about "spaces $X_{i}, i=1,2, \ldots$, any two of them having only a fixed point $b$ in common." 
Details of all this can be found in [6], [8].

We have the following isomorphism theorem:

6.6. THEOREM. On the category $\underline{P}_{0}$ there exists an isomorphism $\alpha: \varepsilon_{*}$ $\approx \mathrm{BU}_{*}$ which allows a unique extension to an isomorphism

$$
\bar{\alpha}: \varepsilon_{*} \approx \overline{\mathrm{BU}}_{*} \text { on } \mathrm{Com}^{f} .
$$

Proof. The existence of an isomorphism $\alpha$ on $\underline{P}_{0}$ is the content of Theorem 7.7 in [6]. Both functors $\varepsilon_{*}$ and $\overline{\mathrm{BU}}_{*}$ are homology theories on $\mathrm{Com}^{f}$ in the sense of Definition 5.1: In the case of $\varepsilon_{*}$ this has been readily proved in [6]. As far as $\overline{\mathrm{BU}}_{*}$ is concerned, this follows from 4.4 and 5.2. Hence we can apply Theorem 5.7, establishing the desired conclusion.

As a corollary we obtain as a consequence of 5.8:

6.7. CoRollary. The homology theory $\varepsilon_{*}: \operatorname{Com}_{h}^{f} \rightarrow \mathrm{Ab}^{\mathrm{Z}}$ allows an

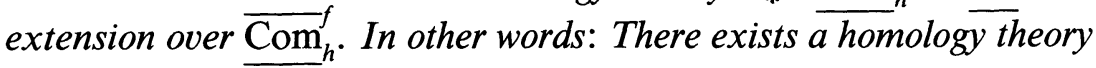

$$
\bar{\varepsilon}_{*}: \overline{\operatorname{Com}}_{h}^{f} \rightarrow \underline{\mathrm{Ab}}^{Z}
$$

such that

$$
\bar{\varepsilon}_{*} h=\varepsilon_{*} .
$$

REMARK. (1) Corollary 6.7 allows of course a direct proof, which uses only the explicit definition of Ext.

(2) Theorems 6.6 and 6.7 give a precise meaning to the statement that " $\varepsilon_{*}$ is complex $K$-homology in the strong shape category". Moreover it gives an explanation to the fact stated in [9], Corollary 5.10, that $\varepsilon_{*}(X) \approx$ $\varepsilon_{*}(Y)$ for two spaces $X, Y$ in $\mathrm{Com}^{f}$ having the same (Borsuk-)shape: Because $\varepsilon_{*}$ is an invariant of strong shape theory, we conclude that $\varepsilon_{*}(X) \approx \varepsilon_{*}(Y)$ for spaces $X, Y$ being equivalent in $\overline{\operatorname{Com}}_{h}$. However two compact metric spaces $X, Y$ are equivalent in $\overline{\mathrm{Com}}_{h}$ if and only if they are equivalent in the Borsuk shape category.

(3) The restriction to finite dimensional compact metric spaces in 6.6 stems from 4.4 which we were not able to establish without that additional assumption. The same restriction is imposed on the corresponding statementy in [8]. Whether Theorem 6.6 is valid for Com (instead of merely $\mathrm{Com}^{f}$ ) is an open question.

7. Survey of the shape construction. This section is not devoted to a rigorous treatment of the strong shape category $\underline{\bar{K}}$ resp. the related homotopy category $\underline{K}_{h}$. This can be found in [4]. We only intend to record 
some facts without proofs in order to make this paper as independent as possible. A different approach to strong shape theory can be found in [7].

(1) A 2-category $\underline{K}$ is an ordinary category (i.e. a 1-category) such that $\underline{K}(X, Y), X, Y \in \mathrm{ob} \underline{K}$, carries again the structure of a category (whose morphism are called 2-morphisms).

A 2-functor $\Phi: \underline{K} \rightarrow \underline{L}$ between two 2-categories is a functor up to 2-morphisms between the underlying 1-categories (this means e.g. that one has only a 2-morphism $\omega: \Phi(g f) \rightarrow \Phi(g) \Phi(f)$, whenever the compositions are defined, which is not necesesarily the identity). These definitions are completed by requiring various compatibility conditions.

By the way, the concept of a $n$-functor (resp. a $n$-category) can be established inductively.

In practice we work with categories of topological spaces, where the 3-category structure can be introduced by taking homotopies as 2-morphisms and homotopies between homotopies as 3-morphisms. Let $H_{0}, H_{1}$ : $X \times I \rightarrow Y$ be two homotopies between mappings $f_{0}, f_{1}$, then a homotopy between homotopies $H_{0}, H_{1}$ is a mapping

$$
\xi: X \times I \times I \rightarrow Y
$$

having the properties:

$$
\begin{aligned}
& \xi(x, t, i)=H_{i}(x, t), \\
& \xi(x, i, s)=f_{i}(s), \quad i=0,1 .
\end{aligned}
$$

In order to equip $\underline{K}(X, Y)$ with the structure of a 2-category, one has to adjust a little the concept of a homotopy; a point, which among other things, we are not going to explain, referring to [4] for the details.

(2) Let $\underline{K}$ be a category of topological spaces (e.g. $\underline{K}=$ Top, $\underline{K}$ $=$ Com, $\underline{K}=$ based or unbased metric spaces and continuous maps) and $\underline{P}$ a subcategory of "good" spaces (e.g. all ANE spaces, all CW-spaces, all compact $\mathrm{CW}$-spaces etc.) The strong shape category $\underline{\bar{K}}$ depends upon the particular choice of this $\underline{P}$; it has the same objects as $\underline{K}$.

Let $X \in \underline{K}$ be any space, then we have a 2-category $\underline{P}_{X}$ having (1) mappings $g: X \rightarrow P \in \underline{P}$ as objects, (2) pairs $(r, \omega): g_{1} \rightarrow g_{2}$ as morphisms, where $r \in \underline{P}$ is continuous and $\omega: r_{1} \approx g_{2}$ a given homotopy.

A 2-morphism $(\nu, \xi):\left(r_{1}, \omega_{1}\right) \approx\left(r_{2}, \omega_{2}\right): g_{1} \rightarrow g_{2}$ consists of a pair where $\nu: r_{1} \simeq r_{2}$ is an ordinary homotopy and $\xi: \omega_{2} \circ \nu g_{1} \simeq \omega_{1}$ a 2-homotopy. The latter is not simply a homotopy between homotopies, but a homotopy class of such a homotopy. This in turn requires the definition of a 3-homotopy which is established analogously to that of a 2-homotopy. 
A 2-functor $\bar{f}: \underline{P}_{Y} \rightarrow \underline{P}_{X}, X, Y \in \underline{K}$ is defined to be a shape mapping $\bar{f} \in \underline{\bar{K}}(X, Y)$ whenever the following conditions are fulfilled:

$\bar{f} 1) \mathrm{g}: Y \rightarrow P \Rightarrow \bar{f}(g): X \rightarrow P$

$\bar{f} 2)(r, \omega): g_{1} \rightarrow g_{2} \Rightarrow \bar{f}(r, \omega)=\left(r, \omega_{1}\right)$ for suitable homotopy $\omega_{1}$.

$\bar{f} 3)$ A corresponding condition involving the 2-morphisms in $\underline{P}_{Y}$. Moreover we require that for a 2-morphism $(\nu, \xi):\left(r_{1}, \omega_{1}\right) \approx\left(r_{2}, \omega_{2}\right)$, $\left(r_{i}, \omega_{i}\right): g_{1} \rightarrow g_{2}$ with $g_{i}=b_{i} a, a \in \underline{P}_{Y}, \xi=\xi^{\prime} a, \bar{f}\left(b_{i}, 1\right)=\left(b_{i}, \gamma_{i}\right)$ one has $\bar{f}(\nu, \xi)=(\nu, \tilde{\xi})$ with

$$
\tilde{\xi}=\delta_{2}^{-1} \circ\left(\gamma_{2}\left(\xi^{\prime} \bar{f}(a)\right)\right)\left(r_{1} \gamma_{1}\right)^{-1} \delta_{1}
$$

and with 2 homotopies

$$
\delta_{i}: \tilde{\omega}_{1} \approx \gamma_{2}\left(\omega_{i}^{\prime} \bar{f}(a)\right)\left(r_{1} \gamma_{1}\right)^{-1},
$$

$\left(\bar{f}\left(r_{i}, \omega_{i}\right)=\left(r_{i}, \tilde{\omega}_{i}\right), \omega_{i}=\omega_{i}^{\prime} a\right)$ being explained before.

Finally we require

f4) For any $\left(r_{1}, \omega_{1}\right) \in \underline{P}_{Y}\left(g_{1}, g_{2}\right),\left(r_{2}, \omega_{2}\right) \in \underline{P}_{Y}\left(g_{2}, g_{3}\right)$. The connecting 2-morphism (whose existence is required in the definition of a 2-functor) is of the form

$$
(1, \eta): \bar{f}\left(\left(r_{1}, \omega_{1}\right)\left(r_{2}, \omega_{2}\right)\right) \rightarrow \bar{f}\left(r_{1}, \omega_{1}\right) \bar{f}\left(r_{2}, \omega_{2}\right) .
$$

Suppose now that $(r, \omega): g_{1} \rightarrow g_{2}$ is a given 1-morphism such that $\omega=\omega^{\prime} a, g_{i}=b_{i} a$, then we have a 2-morphism

$$
(\omega, 1):(r, \omega)\left(b_{1}, 1\right) \rightarrow\left(b_{2}, 1\right)
$$

and a connecting morphism

$$
(1, \eta)^{-1}: \bar{f}(r, \omega) \bar{f}\left(b_{1}, 1\right) \rightarrow \bar{f}\left((r, \omega)\left(b_{1}, 1\right) .\right.
$$

The composition of these 2-morphisms yields a 2-morphism

$$
(r, \tilde{\omega})=\bar{f}(r, \omega) \stackrel{\approx}{\rightarrow} \bar{f}\left(b_{2}, 1\right) \bar{f}\left(b_{1}, 1\right)^{-1}
$$

whose second component $\delta$ is recognized as a 2-homotopy between $\tilde{\omega}$ and $\gamma_{2}\left(\omega^{\prime} \bar{f}(a)\right)\left(r_{1} \gamma_{1}\right)^{-1}$ (using the previous notation $\bar{f}\left(b_{i}, 1\right)=\left(b_{i}, \gamma_{i}\right)$ ).

REMARK. This is a 2-stage strong shape category because it involves only 2-morphisms (and therefore 2-homotopies but no 3-, 4- etc. homotpies. For compact metric spaces this turns out to be sufficient, while for more general spaces one has to go over to $\infty$-categories $\underline{P}_{Y}$ and $\infty$-functors. These objects are much more involved: In case of a $\infty$-functor one does not only have connecting morphisms $\Phi(g f) \rightarrow \Phi(g) \Phi(f)$ but also morphisms regulating non existing associativities on all levels. 
A model for a $\infty$-stage strong shape category (in fact: The homotopy category of such a category) has recently been proposed by Ju. Lisica and S. Mardesic respectively (generalizing the considerations in [4]) independently by the present author at the Leningrad topological conference.

Like in $\underline{K}$ the homotopy category $\underline{\bar{K}}_{h}$ is established by means of mappings $\bar{F} \in \underline{K}(X \times I, Y)$.

We have a functor $h: \underline{K} \rightarrow \underline{\bar{K}}$ being defined by

$$
h(X)=X, \quad X \in \underline{K}
$$

on the objects and by

$$
h(f)(g)=g f
$$

for a morphism $f \in \underline{K}(X, Y)$. Moreover for $Y \in \underline{P}$ we have an assignment

$$
h^{\prime}: \underline{\bar{K}}(X, Y) \rightarrow \underline{K}(X, Y)
$$

defined by

$$
h^{\prime}(\bar{f})=\bar{f}\left(1_{Y}\right)
$$

such that the following properties are satisfied:

$$
\begin{array}{ll}
h^{\prime} h f=f, & f \in \underline{K}(X, Y), \quad Y \in \underline{P}, \\
h h^{\prime} \bar{f} \simeq \bar{f}, & \bar{f} \in \underline{\bar{K}}(X, Y), \quad Y \in \underline{P},
\end{array}
$$

together with corresponding relations for the higher morphisms. We need the following conclusion:

7.1. Proposition. Let $\bar{f} \in \underline{\bar{K}}(X, Y)$ be any shape morphism for $Y \in \underline{P}$, then there exists a continuous $f \in \underline{K}(X, Y)$ such that $h(f) \simeq \bar{f}$.

Proof. Set $f=h^{\prime}(\bar{f})$.

(3) In order to define homology in $\underline{\bar{K}}$ we need a new smash-product $X \bar{\wedge} Y$ for $X, Y \in \underline{K}$, which actually leads us out of the category $\underline{\bar{K}}$ : The new smash-product $X \bar{\wedge} Y$ of two based spaces $\left(X, x_{0}\right),\left(Y, y_{0}\right)$ is a 2-category $\underline{P}_{X} \bar{\wedge} \underline{P}_{Y}$ whose objects are factorizations

$$
\left(X \wedge \underset{g_{1} \wedge g_{2}}{\rightarrow} P_{1} \wedge P_{2} \underset{r}{\rightarrow} P\right)
$$

of objects $(g: X \wedge Y \rightarrow P) \in \underline{P}_{X \wedge Y}$, with $P_{i} \in \underline{P}$. The 1- and 2-morphisms are defined similarly.

One can extend the concept of a shape mapping $\bar{f}: X \rightarrow Y$ to that of a shape mapping

$$
\bar{f}: X_{1} \bar{\wedge} \cdots \bar{\wedge} X_{n} \rightarrow Y_{1} \bar{\wedge} \cdots \bar{\wedge} Y_{m}
$$


as being a 2-functor (more precisely: an equivalence class of such 2-functors)

$$
\bar{f}: \underline{P}_{Y_{1}} \bar{\wedge} \cdots \bar{\wedge} \underline{P}_{Y_{m}} \rightarrow \underline{P}_{X_{1}} \bar{\wedge} \cdots \bar{\wedge} \underline{P}_{X_{n}}
$$

which fulfills the conditions $\bar{f}(1)-\bar{f}(3)$, adapted to the new situation. Details can be found in [4].

Let $\underline{E}$ be any $\mathrm{CW}$-spectrum, then we can define homology groups with coefficients in $\underline{E}$ by

$$
\underline{E}_{n}(X)=\lim _{\rightarrow} \bar{K}_{h}\left(S^{n+l}, E_{l} \wedge X\right), \quad n \in Z .
$$

In [4] $\$ 3$ we explain, among other things, that for compact metric $X$ this $\bar{E}_{n}($ ) is in fact a homology functor which fulfills the Eilenberg-Steenrod axioms for a generalized, reduced homology theory ([4] Theorem 3.1). The crucial problem is embodied in the construction of induced morphisms $\underline{E}_{*}(\bar{f}), \bar{f} \in \underline{\bar{K}}(X, Y)$, which, in turn, requires the existence of a $\bar{f} \wedge 1_{Z}$ : $X \wedge Z \rightarrow Y \wedge Z$. According to [4] Propositions 2.1 this can be accomplished for a $C W$-space $Z$ and a metric space $Y$ (or, alternatively, for all $X, Y, Z$ and continuous $f \in \underline{K}$ ).

We have to refer to the techniques for the explicit construction of a shape mapping, laid down in [4]. In our case it suffices to consider a $\bar{f}$ : $S^{n+l} \rightarrow E_{l} \wedge X$, where $\underline{E}=\left\{E_{l}\right\}$ is a CW-spectrum (which implies, that all $E_{i}$ are "good" spaces in $\underline{P}$ ) and where $X$ is supposed to be a compact metric space. We embed $X$ into a Hilbert cube $Q$ and consider a decreasing sequence of compact ANEs: $Q=P_{1} \supset P_{2} \supset \cdots$, with $\cap P_{i}=$ $X$. It turns out to be sufficient to evaluate $\bar{f}$ only on mappings $1_{E_{l}} \wedge g_{i}$ : $E_{l} \wedge X \rightarrow E_{l} \wedge P_{i}$, where $g_{i}: X \rightarrow P_{i}$ is the inclusion. This is a consequence of [4] Proposition A7. We need this for example in the course of the proof of Proposition 4.1.

At several occasions we use the shape singular complex $\bar{S}(X)$ (resp. $S(X \bar{\wedge} Y)$ ) of a topological space $X \in \underline{K}$ (resp. of a product $X \bar{\wedge} Y$ ). This is defined in complete analogy to the classical case as a simplicial set whose simplexes are shape mappings $\bar{\sigma}^{n} \in \underline{\bar{K}}\left(\left(\Delta^{n}\right)^{+},\left(X, x_{0}\right)\right)$ where $\Delta^{n}$ denotes the standard simplex. The boundary and degeneracy operators are defined like those for the ordinary singular complex $S(X)$. We have that

$$
\bar{S}: \underline{\bar{K}} \rightarrow \mathfrak{\subseteq}_{E} \quad \text { (category of Kan-complexes) }
$$

is a functor,which appears (as in ordinary topology) together with a natural transformation

$$
\bar{\omega}_{X}:|\bar{S}(X)| \rightarrow X, \quad X \in \underline{K}
$$


where $|\cdots|$ denotes, as usual, the geometric realization. We need the fact that:

7.2. Proposition ([2] Theorem 5.1(c)). The shape mapping $\bar{\omega}_{X}$ is a weak homotopy equivalence (hence it induces an isomorphism $\bar{\pi}_{*}\left(\bar{\omega}_{X}\right), \bar{\pi}_{*}$ being the shape homotopy group functor, $\left.\bar{\pi}_{n}\left(X, x_{0}\right)=\underline{K}_{h}\left(S^{n}, X\right)\right)$.

All spaces are supposed to be equipped with a basepoint, all mappings and homotopies are assumed to be base point preserving, although for the mere definition of $\underline{\bar{K}}$ this is not explicitly needed.

When talking about $\underline{K}, \underline{\bar{K}}$ etc. in $\S 1-\S 3$, we assume $\underline{K}$ to be the category $\operatorname{Top}_{0}$. Later on we have to restrict ourselves to the full subcategories Com (= category of compact metric (which always means of course: metrizable) spaces) or even to $\mathrm{Com}^{f}$ ( = category of finite dimensional spaces in Com). In the latter case, every $X \in \operatorname{Com}^{f}$ can already be embedded in some sphere $S^{N}$ and we can assume that all $P_{i} \in \underline{P}$ occurring in the preceding remark are already lying in this $S^{N}$.

As we mentioned already there are many approaches to strong shape theory for compacta (cf. [7]), all of them turn out to be equivalent as homotopy categories (cf. [11], also for further references). The preceding construction leads to individual mappings (rather than to homotopy classes right-away). However it turns out to be a matter of convenience and taste what particular construction somebody is using in order to accomplish a given aim.

Concerning the different kinds of homology theories which appeared in literature, Theorem 5.7 assures us that the homology theory in [7] and the homology theory ${ }^{s} h_{*}$ of [8] are isomorphic: They are both satisfying the Milnor axioms and they agree on finite $\mathrm{CW}$ spaces. The existence of a non-canonical isomorphisms between these homology theories has already been mentioned in [7] $§ 8$.

Finally we must point out that for a CW-spectrum $\underline{E}=\left\{E_{i}\right\}$ we understand the cohomology $\underline{E}^{n}(X)$ as $\lim _{\rightarrow}\left[\Sigma^{k} X, E_{n+k}\right]$ and not in the sense of the Boardman category [1]. This definition is for our purposes more adequate because it corresponds to Čech cohomology (for $\underline{E}$ $=\underline{K(G))}($ see [3], [13]).

\section{REFERENCES}

[1] F. Adams, Stable homotopy and generalized homology, Chicago Lecture Notes in Mathem. The Univ. of Chicago Press (1974).

[2] F. W. Bauer, A shape theory with singular homology, Pacific J. Math., 62 (1976), $25-65$. 
Under what conditions are shape homology $\bar{E}_{*}$ and Steenrod homology ${ }^{s} \underline{E}_{*}$ isomorphic? Shape Theory and Geometric Topology Proceedings, Dubrovnik 1981 Lecture Notes in Math., Vol. 870.

[4] Duality in manifolds, Annali di Matematica Pura ed Applicata (IV) (XXXV), (1984), 241-302.

[5] L. G. Brown, Characterizing $\operatorname{Ext}(X)$ " $K$-Theory and Operator Algebras" Athens, Georgia, Lecture Notes in Math., 575 (1975), 10-18.

[6] L. G. Brown, R. G. Douglas and P. A. Fillmore, Extensions of $C^{*}$-algebras and K-homology, Ann. of Math., (2) 105 (1977), 265-324.

[7] D. A. Edwards and H. M. Hastings, Čech and Steenrod Homotopy Theories with Applications to Geometric Topology, Lecture Notes in Math., 542 (1976).

[8] D. S. Kahn, J. Kaminker and C. Schochet, Generalized homology theories on compact metric spaces, Michigan Math. J., 24 (1977), 203-224.

[9] J. Kaminker and C. Schochet, K-theory and Steenrod homology; Applications to the Brown-Douglas-Fillmore theory of operator algebras, Trans. Amer. Math. Soc., 227 (1977), 63-107.

[10] M. Kernchen, Bemerkungen zur Borel-Moore-Homologie, Manuscripta Math., 39 (1982), 111-118.

[11] Ju. Lisica, Strong theory of shapes and Steenrod-Sitnikov Homology, Sib. Math. J., 24 No. 4, (1983), 557-572.

[12] J. Milnor, On the Steenrod homology theory, Berkeley, (unpublished manuscript) (1960).

[13] K. Morita, Cech cohomology and covering dimension for topological spaces, Fund. Math., LXXXVII (1975), 31-51.

Received November 22, 1985.

JOHANN WOLFGANG GOETHE UNIVERSITAT

ROBERT MAYER STRASSE 6-10

600 FrANKFURT AM MAIN 11

WEST GERMANY 



\section{PACIFIC JOURNAL OF MATHEMATICS EDITORS}

\author{
V. S. VARADARAJAN \\ (Managing Editor) \\ University of California \\ Los Angeles, CA 90024 \\ HERBERT Clemens \\ University of Utah \\ Salt Lake City, UT 84112 \\ R. FINN \\ Stanford University \\ Stanford, CA 94305
}

HERMANN FLASCHKA

University of Arizona

Tucson, AZ 85721

RAMESH A. GANGOLLI

University of Washington

Seattle, WA 98195

VAughan F. R. JONES

University of California

Berkeley, CA 94720

ROBION KIRBY

University of California

Berkeley, CA 94720
C. C. MoOrE

University of California

Berkeley, CA 94720

H. SAMELSON

Stanford University

Stanford, CA 94305

HAROLD STARK

University of California, San Diego

La Jolla, CA 92093

\section{ASSOCIATE EDITORS}
R. AREnS
E. F. BECKENBACH
B. H. NEUMANN
F. WOLF
K. YOSHIDA (1906-1982)

\section{SUPPORTING INSTITUTIONS}

UNIVERSITY OF ARIZONA

UNIVERSITY OF BRITISH COLUMBIA

CALIFORNIA INSTITUTE OF TECHNOLOGY

UNIVERSITY OF CALIFORNIA

MONTANA STATE UNIVERSITY

UNIVERSITY OF NEVADA, RENO

NEW MEXICO STATE UNIVERSITY

OREGON STATE UNIVERSITY
UNIVERSITY OF OREGON UNIVERSITY OF SOUTHERN CALIFORNIA STANFORD UNIVERSITY UNIVERSITY OF HAWAII UNIVERSITY OF TOKYO UNIVERSITY OF UTAH WASHINGTON STATE UNIVERSITY UNIVERSITY OF WASHINGTON 


\section{Pacific Journal of Mathematics}

\section{Vol. 128, No. $1 \quad$ March, 1987}

Anthony Peter Bahri and Peter Gilkey, The eta invariant, $\operatorname{Pin}^{c}$ bordism, and equivariant $\operatorname{Spin}^{c}$ bordism for cyclic 2-groups $\ldots \ldots \ldots \ldots \ldots \ldots 1$

Friedrich-Wilhelm Bauer, Extensions of generalized homology theories . . . 25

Marilyn Breen, A characterization theorem for compact unions of two

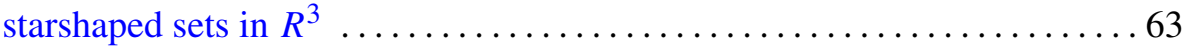

Boniface Ihemotuonye Eke, Special generating sets of purely inseparable extension fields of unbounded exponent $\ldots \ldots \ldots \ldots \ldots \ldots \ldots \ldots \ldots \ldots$

Robert William Gilmer, Jr. and William James Heinzer, Jónsson $\omega_{0}$-generated algebraic field extensions $\ldots \ldots \ldots \ldots \ldots \ldots \ldots \ldots \ldots . . .61$

Guido Lupacciolu, Holomorphic continuation in several complex variables

Douglas C. McMahon, Jaap C. S. P. van der Woude and Ta-Sun Wu,

Connectedness related to almost periodicity of compositions of flow

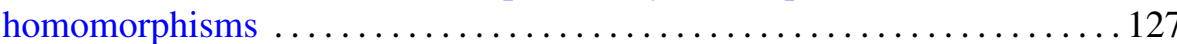

Sophocles Mercourakis, Some characterizations of analytic metric spaces

Peter Frederick Stiller, The Picard numbers of elliptic surfaces with many

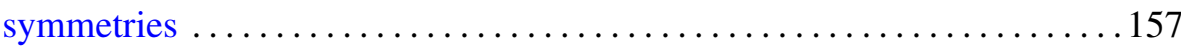

David J. Winter, Reducible complements of Lie algebra radicals ........ 191 Rade Živaljević, On a cohomology theory based on hyperfinite sums of microsimplexes 